\title{
Brain (hyper)excitability revealed by optimal electrical stimulation of GABAergic interneurons
}

\author{
F. WENDLING, PhD ${ }^{1,2,{ }^{*}}$, U. GERBER, MD, PhD ${ }^{3}$, D. COSANDIER-RIMELE, PhD ${ }^{2}$, A. NICA, MD ${ }^{4}$, J. DE \\ MONTIGNY, MSC ${ }^{1,2}$, O. RAINETEAU, PhD ${ }^{3}$, S. KALITZIN, PhD ${ }^{5}$, F. LOPES DA SILVA MD, PhD ${ }^{6}, \mathrm{P}$. \\ BENQUET, PhD ${ }^{1,2}$
}

\section{ABSTRACT}

Background. Neurological disorders are often characterized by an excessive and prolonged imbalance between neural excitatory and inhibitory processes. A ubiquitous finding among these disorders is the disrupted function of inhibitory GABAergic interneurons.

Objective. The objective is to propose a novel stimulation procedure able to evaluate the efficacy of inhibition imposed by GABAergic interneurons onto pyramidal cells from evoked responses observed in local field potentials (LFPs).

Methods. Using a computational modeling approach combined with in vivo and in vitro electrophysiological recordings, we analyzed the impact of electrical extracellular local bipolar stimulation (ELBS) on brain tissue. We implemented the ELBS effects in a neuronal population model in which we can tune the excitationinhibition ratio and we investigated stimulation-related parameters. Computer simulations led to sharp predictions regarding: i) the shape of evoked responses as observed in local field potentials, ii) the type of cells (pyramidal neurons and interneurons) contributing to these field responses and iii) the optimal tuning of stimulation parameters (intensity and frequency) to evoke meaningful responses. These predictions were tested in vivo (mouse). Neurobiological mechanisms were assessed in vitro (hippocampal slices).

Results. Appropriately-tuned ELBS allows for preferential activation of GABAergic interneurons. A quantitative Neural Network Excitability Index (NNEI) is proposed. It is computed from stimulation-induced responses as reflected in local field potentials. NNEI was used in four patients with focal epilepsy. Results show that it can readily reveal hyperexcitable brain regions.

Conclusion. Well-tuned ELBS and NNEI can be used to locally probe brain regions and quantify the (hyper)excitability of the underlying brain tissue.

Keywords: neurostimulation, electrical, extracellular bipolar direct stimulation, neuronal network, excitability, GABAergic interneuron, local field potential, depth-EEG, computational model, in vivo, in vitro, epilepsy

Acronyms: ELBS: extracellular local bipolar stimulation; LFP: local field potential; EEG: electroencephalography, SEEG: stereo-EEG; NMM: neural mass model; NNEI: neuronal network excitability index; PSP: post-synaptic potential; EPSP: excitatory PSP; IPSP: inhibitory PSP. 


\section{Introduction}

2 Normal brain functions require input from local GABAergic interneurons onto pyramidal cells to

3 maintain a balance between excitatory and inhibitory processes in cortical circuits [1]. This balance is

4 of utmost importance and many neurological disorders are characterized by a disrupted function of

5 GABAergic interneurons leading to impaired neuronal discharge and the associated symptomatology

6 (see review in [2]). This dysregulation can be either diffuse as in autism spectrum disorders [3], [4],

7 Down syndrome [5], and mood disorders [6] or more focal as in chronic pain [7] and epilepsy [8], as demonstrated in animal models for these diseases.

In this context, a diagnostic procedure that would provide specific information about the integrity of inhibitory interneuron functions in the brain would be highly beneficial. Neurostimulation-based methods constitute an appealing option since evoked brain responses convey relevant information regarding the underlying excitatory and inhibitory neuronal processes responsible for their generation. In this perspective, three main challenging issues must be addressed. First, what are the optimal stimulation parameters (intensity, frequency, polarity) for which a reliable diagnostic can be achieved? This has always been - and still is - a matter of debate for applications in neurological disorders in general [9], and in epilepsy in particular [10]. Second, how do responses induced by stimulation and observed in local field potentials (LFPs) relate to the interneuron function in the stimulated brain tissue? Third, how can unequivocal information be extracted from LFP responses in order to quantitatively measure the excitability state of the local neuronal networks?

In this study, these three issues are dealt with. Following upon pioneering studies where active stimulation paradigms were used in order to detect the seizure onset zone and, eventually, to anticipate epileptic seizures $[11,12][13]$, the objective is to design an invasive electrical stimulation procedure that can provide quantitative information about the excitability level of locally-stimulated neural networks. 
1 Using a computational modelling approach [14] combined with in vivo and in vitro recordings [15],

2 we show that extracellular, local, bipolar stimulation (ELBS) can mainly activate GABAergic 3 interneurons provided that intensity and frequency parameters are appropriately tuned. We disentangle the network and cellular mechanisms involved in evoked responses and provide guidelines for optimal adjustment of the stimulation parameters. Finally, we propose a Neural Network Excitability Index (NNEI) that can be computed from responses recorded in LFPs. Regarding the clinical application, results obtained in four patients with drug-resistant focal epilepsy indicate that NNEI can be used to identify hyperexcitable brain regions. Considering the central role of GABAergic interneurons in the brain [16], this study opens numerous perspectives such as following disease progress.

\section{Materials and methods}

In the following, the proposed stimulation procedure is referred to as Extracellular Local Bipolar Stimulation (ELBS).

\section{Computational modeling and predictions about ELBS effects on a population of neurons}

The effects of ELBS were first assessed using a computational modelling approach. The different steps of this investigation are summarized in Fig.1-A. A neural mass model (NMM, neuronal population level) was used to simulate LFPs in response to ELBS. This model accounts for the coupling between the electric field resulting from stimulation and the average membrane at the two sub-populations of PCs and INs. In addition, the excitability level of the local population of neurons could be tuned by adjusting the amplitude of average excitatory and inhibitory postsynaptic potentials. From simulated LFP signals, the Neural Network Excitability Index was computed and plotted versus the model excitability. From with comparison, we could make predictions i) about the contribution of PC and INs to evoked responses and ii) about optimal ELBS intensity and frequency values for which the NNEI value (range: $[0,1])$ best reflects the excitability level (low, medium, high). 
1 In the following, details about the NMM, the coupling between NMM and stimulation, the simulated

2 field potentials and the NNEI metric are provided.

Neural mass model (NMM). The computational model is inspired from neurophysiology and consists in a neuronal population comprising two sub-populations of neurons, namely PCs and INs. Although this model is aggregated (no explicit representation of individual neurons), it offers three main advantages. As directly inspired from neurophysiology, it has been shown to provide relevant insights into the relationship between excitation-/inhibition-related model parameters, on the one hand, and the field activity as recorded by extracellular electrodes, on the other hand. Readers may refer to [17-20] for detailed information about the model (structure, equations, parameter interpretation and setting). In brief, as shown in Fig.1-B, the design is intended to macroscopically represent a simplified neuronal network consisting of two synaptically coupled subsets: pyramidal cells (PCs) and local interneurons (INs). In each subset, a linear transfer function $h_{e}(t)=A a t e^{-a t}$ or $h_{i}(t)=B b t e^{-b t}$ relates the average pre-synaptic pulse density of action potentials to an average post-synaptic membrane potential, either excitatory (EPSP) or inhibitory (IPSP). In turn, a nonlinear function (asymmetric sigmoid function $\left.S(v)=\frac{2 e_{0}}{1+e^{r\left(v_{0}-v\right)}}\right)$ accounts for threshold and saturation effects in the relation between the average post-synaptic potential and the average density of pulses fired by the neurons.

Coupling between NMM and stimulation. To mimic ELBS effects, we introduced, in the model, the impact of biphasic current pulses applied locally with extracellular bipolar electrodes (Fig.1-B). Regarding the coupling model, we considered that i) each stimulation pulse induces a transient change in the mean membrane potential of both PC and IN subsets [21] and ii) this perturbation of the membrane potential is a linear function of the externally-applied current magnitude [13]. In practice, as illustrated in Fig.1-C, periodic stimulation pulses denoted by $p(t)$ were thus added to the mean membrane potential at the input of each sigmoid function $S(v)$. Thus, according to this coupling model, and since pulses are biphasic, a transient increase of the average firing rate in 
1 stimulated neuron subsets is systematically achieved (output of each sigmoid function $S(v)$ ), in line

2 with experimental data [22]. The above considerations lead to the $6^{\text {th }}$-order set of ordinary

3 differential equations that govern the temporal dynamics in the model:

$\mid \begin{aligned} & \dot{y}_{0}(t)=y_{3}(t) \\ & \dot{y}_{3}(t)=A a S\left(y_{1}-y_{2}+p(t)\right)-2 a y_{3}(t)-a^{2} y_{0}(t) \\ & \dot{y}_{1}(t)=y_{4}(t) \\ & \dot{y}_{4}(t)=A a\left\{n(t)+C_{2} S\left[C_{1} y_{0}(t)+p(t)\right]\right\}-2 a y_{4}(t)-a^{2} y_{1}(t) \\ & \dot{y}_{2}(t)=y_{5}(t) \\ & \dot{y}_{5}(t)=B b\left\{C_{4} S\left[C_{3} y_{0}(t)+p(t)\right]\right\}-2 b y_{5}(t)-b^{2} y_{2}(t)\end{aligned}$

5 where parameters $A$ and $B$ denote the amplitude of average EPSPs and IPSPs, respectively, where $a$

6 and $b$ (expressed in $\mathrm{s}^{-1}$ ) are used to adjust the rise and decay time of these PSPs and where

7 parameters $\mathrm{C} 1-\mathrm{C} 4$ represent the average number of synaptic contacts between PC and IN sub-

8 populations. Note that parameters $A$ and $B$ are used to control the level of excitability in the model.

9 This set of equation is solved by numerical integration methods (Euler, fixed step method, for example). Special attention must be paid to its stochastic nature due to the noise input $n(t)$.

Simulated local field potentials. The average postsynaptic activity of the PC subset was chosen as the model output, according to the assumption that summated PSPs at the level of pyramidal cells constitute the main contribution of LFPs. This model output (see example in Fig.1-C) was compared to actual LFPs recorded in vivo, both in mice and in patients.

Neural Network Excitability Index. Details about the NNEI computation are provided in the Supplementary Material 1 (SM1). In brief, the NNEl is a normalized quantity (ranging from 0 to 1 ) that measures the phase similarity among signals. It discloses low values where LFP evoked responses (time locked to each pulse of the stimulation train) have similar time-courses, and high values where LFPs have irregular time-courses, provided that the stimulation intensity is appropriately tuned. 
1 In vivo experiments

2

In vitro experiments 7.2 with $0.2 \%$ Biocytin

\section{Clinical data}

In vivo experiments (LFP recordings and ELBS) were performed in adult C57BL/6RJ male mice $(80 \pm 5$ days, $n=18$ ). Sub-convulsive dose of pentylenetetrazole $(P T Z, 35 \mathrm{mg} / \mathrm{kg}, I P)(D h i r, 2012)$ was used to induce an increase of the level of brain excitability in naive animals. All experiments were in accordance with the guidelines of INSERM for animal care in research, and were approved by the ethics committee of Rennes (agreement $N^{\circ}$ R-2012-PB-OI). See Supplementary Material SM2 for details. Quiet awaking periods were retained for NNEI computation. LFP signals were recorded using a video-EEG monitoring system (Deltamed TM, Natus Group, sampling at $2048 \mathrm{~Hz}$ ). The signals were processed using both MATLAB and C-code routines developed in the lab.

In vitro experiments (patch-clamp and LFP recordings, ELBS) were performed in rat hippocampal organotypic slice cultures [23] that offer the major advantage (as compared with acute slices) that functional properties (electrophysiological and synaptic) of neurons in studied CA1 networks are well preserved [24]. Details are provided in Supplementary Material SM2. Slice cultures were then transferred to a patch-clamp recording setup and superfused with artificial cerebrospinal fluid (ACSF) equilibrated with 95\% O2/5\% CO2 containing (in mM): $124 \mathrm{NaCl}, 2.5 \mathrm{KCl}, 26 \mathrm{NaHCO}, 1.25 \mathrm{NaH} 2 \mathrm{PO} 4$, 10 glucose, $3 \mathrm{CaCl} 2,2 \mathrm{MgCl} 2, \mathrm{pH}$ 7.4. Field and intracellular recordings were simultaneously performed. The patch clamp pipette (4-6 $\mathrm{M} \Omega$ ) was placed in the vicinity of the field recording pipette and filled with $140 \mathrm{~K}$-gluconate, $5 \mathrm{NaCl} 10$ HEPES, 10 phosphocreatine, $1 \mathrm{mM} \mathrm{EGTA}, 1 \mathrm{mM} \mathrm{MgCl} 2 \mathrm{pH}$

The clinical data were recorded in four patients (P1-P4, Table 1) undergoing pre-surgical evaluation of drug-resistant temporal lobe epilepsy (TLE, P1) and temporal 'plus' epilepsies [25] (P2-P4). StereoEEG (SEEG, depth electrodes) recordings were performed during long-term video-EEG monitoring (5 
days, Micromed, SystemPLUS Evolution) using intracerebral multiple lead electrodes placed intracranially according to Talairach's stereotactic method. SEEG was carried out as part of regular clinical care. The location of each electrode contact was post-operatively checked using postimplantation 3D CT-scan and preoperative 3D MRI reconstruction into Medtronic Neuronavigation system. ELBS procedure was performed using an isolated biphasic stimulator (Micromed Inc., Energy Surgical version) allowing for fine tuning around optimal intensity value $(0.1-0.5 \mathrm{~mA})$ and frequency value $(5-10 \mathrm{~Hz})$. Stimulation parameter values used in each patient are given in Table 1 . Patients gave written informed consent about the stimulation procedure that was approved by the local committee of the Neurology department, Rennes University Hospital. During the stimulation session, none of the patients experienced a particular sensation or showed a particular symptom.

\section{Calibration of the Extracellular Local Bipolar Stimulation (ELBS) intensity value}

In the computational model, we determined that the optimal intensity value (i.e. leading to a high contrast in NNEI values associated with low and high excitability states) is obtained when evoked responses start to become discernible in the LFP for repetitive pulses. This finding was translated into a statistical test based on the linear correlation among responses (see Supplementary Material SM3 for theoretical basis). This calibration procedure was applied for in vivo recordings. ELBS was applied starting from a very low intensity ( $1 \mu \mathrm{A}$ in mice, $0.2 \mathrm{~mA}$ in patients) and was gradually increased until a negative wave following the artefact was discernible in the LFP. For each train, the statistical test was performed and $w$ was computed. The intensity for which $w$ reached the 2.6 threshold value was kept (Sup. Mat. SM3). In practice, in mice, the optimal intensity was generally about $1.4 \mu \mathrm{A}$ and the NNEI value around 0.1 under "normal" excitability conditions. It is worth mentioning that each biphasic pulse consisted of a first positive $(100 \mu \mathrm{s})$ pulse followed by a second negative pulse (100 $\mu s)$. The amplitude of the second negative pulse was adjusted by a few tens of nano-amperes (0.01 $0.05 \mu \mathrm{A})$ to compensate the offset between both pulses what resulted in evoked responses very 
marginally contaminated by the stimulation artefact. In patients (Table 1), the optimal intensity was $0.5 \mathrm{~mA}(\mathrm{P} 1)$ and $0.3 \mathrm{~mA}(\mathrm{P} 2-\mathrm{P} 4)$.

The difference between intensity values in mouse and in human is explained by the dramatic difference in the electrode size (microwires vs. clinical macroelectrodes). Indeed, we estimated, as a first approximation, the order of magnitude of the current density produced in both cases. We considered the current density $\vec{J}$ in the space between the two closest electrode contacts (for in vivo electrodes, in the region of space between the tips of the twisted wires; for human electrodes, between the two closest contacts along the stimulation electrodes). We used the general expression $I=\iint \vec{J} . \overrightarrow{d s}$ for the current density, which we simplified as $I=J . S$ where $S$ is the surface of the electrode contact. For in vivo electrodes, the electrode tip (disk) has a diameter $D=139 \mu \mathrm{m}$ (Table 2), which we used to compute the surface $S=\pi\left(\frac{D}{2}\right)^{2}$. The current intensity was $1.4 \mu \mathrm{A}$, resulting in a current density estimation of $92 \mathrm{~A} / \mathrm{m} 2$. For clinical electrodes, each contact is cylindrical, with a diameter $\mathrm{D}=0.8 \mathrm{~mm}$ and a contact height $\mathrm{H}=2 \mathrm{~mm}$ (Table 2), with the contact surface being calculated as $S=\pi$. D. H. The current intensity was on the order of $0.4 \mathrm{~mA}(0.3-0.5$, Table 1$)$, which results in a current density estimation of $79 \mathrm{~A} / \mathrm{m} 2$. Interestingly, this estimation is in the same order of magnitude, confirming that, despite the significant difference in electrode physical properties, the stimulation applied in vivo and in humans operates at comparable scales.

\section{Results}

Since the computational models provided the framework for the experimental studies we start with the former.

Computational model predictions. A key feature of the computational model [18] is the ability to simulate LFPs according to i) varying levels of excitability states and ii) various conditions of stimulation parameters. Changes in excitability in the model, from a low to a high level were obtained by simply changing the ratio between two parameters, namely the average amplitude of 
the EPSP and the IPSP. Therefore we investigated the effects of changing the excitability level both on background (on-going) activity and on simulated LFPs, under two conditions: without stimulation (no stim., i.e. "passive" condition), and with stimulation (stim., i.e. "active" paradigm).

In the first case (Fig. 2A, no stim.), we did not find any noticeable change in the background activity nor in the corresponding power spectra when excitability changed from low to high level. In the second case (Fig. 2B, stim.), the simulated pulse stimulation applied to both pyramidal cells and interneurons, elicited small LFP waves following the stimulation artefacts. In the case of low excitability, the time-course of LFP signals was found to be reproducible from one pulse to another, as shown by surperimposed responses (each time-locked to the pulse onset). In contrast, for high excitability, surperimposed responses were found to have a more irregular, variable time course.

We devised a neural network excitability index $(0<$ NNEI $<1)$ that quantifies the level of the excitability state (for details see Supplementary Material 1 -SM1- Computation of the NNEI). This index displays i) low values when the LFP responses display constant phase w.r.t. the pulse stimulation onsets and ii) high values when these responses show irregular phases.

The NNEI was found to provide a reliable indication of the modelled level of excitability state in the case where both PCs and INs were stimulated (Fig. 2B), and also in the case where only INs were stimulated (Fig. 2C, left). Strikingly, no such consistent responses appeared in the LPF when stimulation was applied only to PCs (Fig. 2C, right). In addition, the NNEI was blind to changes of excitability state (low, medium, high) in this case, suggesting that direct excitation of INs is a necessary condition to evoke discernible consistent responses in the LFP and subsequently to obtain a NNEI value indicative of the excitability level in the model.

Computer simulations allowed us, further, to assess the effects of two crucial stimulation parameters, namely the intensity and the frequency of the bipolar single pulse stimulation. Fig. 2Dleft displays the impact of pulse intensity on simulated responses for 3 excitability levels, low, 
medium and high. The model reveals that both the shape and the phase coherence of evoked LFP responses depend on the stimulation intensity value (I-value). For very low I-values, evoked responses were either absent or of very low amplitude, i.e. simulated signals were difficult to distinguish from background activity. For intermediate I-values, simulated signals disclosed small coherent evoked responses. For high I-values, simulated signals took the form of high amplitude, highly-coherent evoked responses. These intensity effects were quantified for three excitability conditions (Fig. 2D, right). For very low I-values (dark to light blue color), the NNEI was either - blind to - or provided incomplete information on - the level of the excitability condition, as values remained limited to a narrow range $[0.5,0.85]$. Similarly, for high I-values (red to orange color), the NNEI values were restricted also to a narrow interval $[0,0.25]$. In contrast, for intermediate I-values (yellow to blue color), the NNEI range was much broader $([0.05,0.65])$ indicating that a better contrast between low and high excitability conditions was achieved in this case. These modelling results indicate that intensity is a critical parameter for interpretation of NNEI values. Results regarding the frequency parameters are provided in Fig. $2 \mathrm{E}$ and $2 \mathrm{~F}$. They showed that the NNEI is a reliable indicator for the excitability level in the model as long as the stimulation frequency remained below $10 \mathrm{~Hz}$.

Overall, the model shows that stimulation parameters must be "optimally" tuned to obtain responses from which NNEI values are able to differentiate between low and high levels of excitability state: the bipolar stimulation must be applied to local neuronal subpopulations at appropriate intensity (Fig. 2D) and at appropriate frequency (Fig. 2F).

In vivo validation of model predictions. We performed in vivo stimulations in the hippocampus of the mouse while recording LFPs in the CA1 stratum radiatum (see Methods and Sup. Mat. SM2). In order to fine-tune the intensity value, we followed the ELBS calibration procedure described in the methods (see also Sup. Mat. SM3). At "optimal" I-value, small-amplitude evoked responses appeared in the LFPs, as illustrated in Fig. 3B. The responses obtained using the model, in silico, and in vivo are 
shown side-by-side in Fig. 3A and 3B. This provides a direct comparison between in silico and in vivo results for control animals. As depicted, in vivo results strongly resemble those obtained in the computational model, in terms of i) shape of evoked responses vs. I-value, ii) evolution of the w statistics vs. I-value and iii) evolution of the NNEI vs. I-value.

The ability of this fine-tuning procedure to reveal excitability changes in vivo was then evaluated by changing the level of excitability of the mouse hippocampus (Fig. 3C). For an optimally-tuned stimulation intensity I-value $(1.3+/-0.1 \mu \mathrm{A})$, the NNEI dramatically increased just after PTZ injection (0.2 to 0.8 ) (Fig. 3D). It stayed high (NNEI > 0.4) for about $10 \mathrm{~min}$ and then returned to basal value (>0.2) which is compatible with the pharmaco-kinetics of PTZ [26]. It should also be noted that although this low dose of PTZ did not induce seizures $(n=18)$, infrequent sporadic interictal epileptic spikes (IESs, 7/min on average over the 15 min period) were observed. Differences between NNEI values measured before, during and after PTZ injection were found to be significantly different ( $p<0.01$, Fig. 3D, right). Finally, we showed that "non-optimal" tuning of the intensity value leads to ambiguous results, as reported in the supplementary Figure S2. When the same experiment is repeated with non-optimal stimulation intensities (either too low or too high), LFP waveforms, either not distinguishable from background or very pronounced, were also observed, as predicted by the NMM model.

These in vivo results confirm that the fine tuning of both stimulation intensity and frequency parameters is crucial to derive an interpretable NNEI, i.e. a NNEl that reliably reflects the underlying excitability condition.

Cellular mechanisms of ELBS. To resolve the cellular mechanisms underlying the evoked responses observed in LFPs, ELBS was performed in vitro using organotypic hippocampal slices. Simultaneous intra- and extracellular recordings were performed in the CA1 subfield (Fig. 4A) under ELBS. Pyramidal cells (PCs) were identified according to the following criteria: anatomical location (PC 
1 layer), morphology (biocytin labelling), presence of dendritic spines, firing patterns and resting

2 membrane potential (see details in Methods and Supplementary Material SM2).

The ELBS intensity was progressively increased from 10 to $500 \mu \mathrm{A}$. The first suprathreshold LFP response was associated with a hyperpolarization of PCs (for an average stimulation intensity of 116 $\pm 9 \mu \mathrm{A}, \mathrm{n}=36)$ (Fig. 4B). Interestingly, simultaneous extra-/intracellular recordings $(\mathrm{n}=5)$ showed that this minimal stimulation intensity, necessary to elicit a measurable small deflection in the LFP, was systematically associated with an IPSP in PCs.

Under conditions of low excitability (ACSF), repetitive stimulation at $2 \mathrm{~Hz}$ induced simultaneous and reproducible small LFP deflections (extra-cell.) and IPSPs (intra-cell.), without noticeable rundown (Fig. 4C). This suprathreshold stimulation intensity was applied under control (low excitability, ACSF, Fig. 4D top) and high excitability (low $\mathrm{Mg}^{2+}, \mathrm{GABA}_{\mathrm{A}}$ receptor antagonist bicuculline) conditions (Fig. 4D, bottom). For control conditions, field evoked responses were found to be coherent in time and to display very similar time courses (Fig. 4D, upper superimposition of colored traces). Conversely, under conditions of increased excitability, evoked LFP responses were much less synchronized with respect to stimulation onset (Fig. $4 \mathrm{D}$ bottom). Note that under blockade of $\mathrm{GABA}_{\mathrm{A}}$ receptors, their polarity reversed (Fig. 4D). This absence of coherence among LFP responses is reflected in a significant increase in the NNEI (from 0.02 to $0.33, p<0.01, n=5$ )(Fig. 4D right panel), in agreement with in vivo and in silico findings.

The synaptic basis of the observed hyperpolarization was pharmacologically assessed. As illustrated in Fig. 5A, the observed intracellular inhibitory responses were fully blocked by bath application of bicuculline $(10 \mu \mathrm{M})(97 \pm 4 \%, \mathrm{n}=8, \mathrm{p}<0.01)$ or $\operatorname{TTX}(1 \mu \mathrm{M})(98 \pm 2 \%, \mathrm{n}=6, \mathrm{p}<0.01)$. The evoked LFP response decreased under superfusion of bicuculline $(10 \mu \mathrm{M}, \mathrm{n}=6)$. These results indicate ELBSinduced responses reflect the synaptic activation of $G A B A_{A}$ receptors on PCs, suggesting that ELBS promotes the excitation of interneurons and therefore the release of GABA onto targeted PCs. 
1 To assess whether GABA released by ELBS was sufficient to locally inhibit PCs, direct bipolar

2 stimulation was applied during the evoked firing of the pyramidal cell (in current-clamp mode). As

3 depicted in Fig. 5B, in fact ELBS transiently stopped its firing.

4 PSPs on PCs were then measured and plotted against the ELBS intensity values (0 to $400 \mu \mathrm{A})$. At low intensity (100-250 $\mu \mathrm{A})$, IPSPs were recorded (Fig. 5C) in most pyramidal cells (89\%, 32 pyramidal cells out of 36). Conversely, beyond $250 \pm 30 \mu \mathrm{A}$, EPSPs/spike were measured in all recorded PCs $(n=21)$. Therefore, in this latter case, ELBS excited at least a few pyramidal cells. This result indicates that an inhibitory effect is obtained within a restricted range of ELBS values (Fig. 5D). With supra-threshold ELBS $(100-200 \mu A)$, IPSPs were recorded in $89 \%$ of PCs $(n=32)$ whereas higher intensity $(220-400 \mu A)$ stimulation induced EPSPs and/or action potentials in all recorded PCs ( $n=21)$ (Fig. 5E).

Overall, these in vitro results show that LFP small-amplitude responses simulated in silico and observed in vivo correspond to short-term GABAergic inhibition of PCs. Most important, these results suggest that low intensity ELBS allows the preferential activation of GABAergic interneurons versus pyramidal cells.

Clinical application. In drug-resistant epileptic patients candidate to surgery, a pre-surgical evaluation consists in the direct recording of brain activity from multiple intracranial electrodes (Fig. 6 A). We raised the hypothesis that the proposed low intensity ELBS could be performed using these intracranial electrodes in order to assess GABAergic inhibition in healthy and epileptic brain tissue. Prospectively, we took advantage of the unique opportunity that of the 4 patients (Table 1 ) who underwent ELBS, three patients (P1 - P3) had depth-electrodes implanted bilaterally in both hippocampi. In patient P1, the electrical pattern of the left hippocampus (LHIP) strongly suggested hippocampal sclerosis. This was confirmed by the postoperative histology. Fig. 6B illustrates a subclinical epileptic discharge recorded from the LHIP whereas the simultaneous activity in the contralateral hippocampus (RHIP) is normal. Low intensity low frequency ELBS $(0.5 \mathrm{~mA}, 10 \mathrm{~Hz})$ protocol was applied alternatively to the LHIP and in the RHIP (Fig. 6C) during the interictal period. As 
1 in the computational model, in the studies both in vivo and in vitro, ELBS produced small but

2 discernible coherent LFP responses in the healthy RHIP but less regular in the epileptogenic LHIP (Fig.

36 D). This result was substantiated by the mean NNEI values for each train of 50 pulses applied (Fig.

$4 \quad 6 C$ ). As shown in Fig. 6E, significantly higher (Mann Whitney U-test, $p<0.01$ ) NNEI values were found

5 in the LHIP $(0.45+/-0.04)$ compared to the healthy RHIP $(0.15+/-0.03)$.

In patients P2 and P3 (Fig. 7A and 7B), under ELBS $(0.3 \mathrm{~mA}, 5 \mathrm{~Hz})$, high NNEl values $(0.3<\mathrm{NNEI}<0.5)$ were computed from SEEG signals recorded on both sides, indicating increased excitability in both the left and right hippocampus. This abnormal excitability was further confirmed by the presence of locally-generated epileptic spikes that occurred either asynchronously or simultaneously at both sides (Fig. 7A and 7B, right panels). Finally in patient P4 (Fig. 7C), NNEI values, computed from SEEG signals recorded in the right hippocampus (RHIP) and in the right middle temporal gyrus (RMTG) under ELBS $(0.3 \mathrm{~mA}, 5 \mathrm{~Hz})$, were found to be very different at both sites (RHIP: NNEI=0.34, RMTG: NNEI=0.04) suggesting abnormal excitability in the RHIP and healthy tissue in the RMTG. This result was confirmed by electrophysiological data (rhythmic discharges of epileptic spikes occurring in the RHIP with simultaneous normal activity in the RMTG, Fig. 7C, right panel) and by imaging data (MRI strongly evocative of a right hippocampal sclerosis).

\section{Discussion}

Altered brain excitability is a hallmark of many neurological disorders. In particular, abnormal hyperexcitability in neural networks has been demonstrated in most, if not all, types of experimental models of epileptogenesis (review in [27]) as well as in human epileptic tissue [28]. An important challenge, however, is how to assess quantitatively the excitability state of brain tissue in vivo.

To the best of our knowledge, the present study is the first to show i) that appropriately tuned ELBS is capable of preferentially eliciting responses mediated by GABAergic interneurons and ii) that this 
evoked response as reflected in the LFP can be advantageously used to quantify the underlying network excitability.

The proposed stimulation procedure is particularly suited as GABAergic interneurons play a central role in the CNS and as their dysregulation (sometimes referred to as "interneuronopathy" $[29,30]$ was observed in many pathological conditions, including epilepsy ([31,32], among others). In addition, active paradigms (i.e. stimulation-based) have been shown to outperform passive procedures (i.e. based on the observation of spontaneous EEG activity) regarding the capacity to reveal the functional properties of neuronal systems in epilepsy $[12,13,33]$.

To elaborate this stimulation procedure, to understand the effects of induced currents and to define the optimal stimulation parameters (intensity, frequency), we followed a combined in silico/in vivo/in vitro approach.

To our knowledge, the detailed analysis of ELBS effects has never been performed before in a neural mass model. Results indicated that the NMM could accurately reproduce actual LFPs despite a number of limitations regarding the representation of biophysical effects of ELBS. Indeed, the coupling model used in this study is rather simple as limited to a mean membrane voltage change linearly related to the intensity of short-duration (100 $\mu \mathrm{s})$ biphasic pulses. It does not account for the specific features pf electrodes (surface area, curvature of the electrode tip, resistive coating to electrode contacts, ...) that alter the current-density distribution and thus the way local populations of neurons are impacted by non-uniform electric fields [34]. In this respect, results from detailed studies [35] will help to improve the physical realism of the model and possibly lead to calibrated values which remain, in the present form, disconnected from real values. Nevertheless, and in spite of aforementioned limitations, this study shows that the model somehow captures "the essence" of the observed phenomenon into a simple (but not too simple) neural mass representation of i) the dynamic properties of a population of interacting excitatory and inhibitory neurons, ii) the generation of LFPs (major contribution of PSPs) and iii) the direct and transient depolarizing effect of 
1 local stimulation. By aggregating these main "ingredients", some predictions logically followed. First,

2 low excitability means high inhibitory drive onto PCs and thus large IPSPs (4 times > EPSPs) and

3 consequently a reflection in the LFP as a small deflection following stimulation pulses. High

4 excitability means lower inhibitory drive (and increased excitatory drive) resulting from a lesser contribution of IPSPs to the LFP which are, at the same time, masked by the input noise which is excitatory. Combined with experimental data, the model pointed toward the critical effect of three factors: the cell type being stimulated, the stimulation intensity and the stimulation frequency.

Overall, this theoretical/experimental approach led to the following main findings.

Appropriate stimulation intensity is required. The described low intensity ELBS procedure is efficient for revealing the underlying excitability level if, and only if, the current intensity is appropriately adjusted. As shown by in silico and in vivo data, when this intensity value falls within the "optimal" range, then the NNEI value provides a meaningful indication about the excitability of the probed networks. Using in vitro data, we found that this optimal range corresponds to an "inhibitory window" in which IPSPs are preferentially elicited onto pyramidal cells. In this study, we provided a systematic method to define the optimal stimulation intensity based on the statistical correlation among evoked field responses.

Regarding the ELBS frequency, beyond $10 \mathrm{~Hz}$, both computational and in vivo data showed LFP responses that did not lead to a reliable NNEI value. In the neural mass model, this result is explained by nonlinear system dynamics. At high frequency, the system is being constantly stimulated during a transient regime, i.e. during the time-course of its response to pulses. In that case, the NNEI computation is performed on truncated responses, and gives erroneous estimates. The same applies to real data, where responses were also found to be truncated under similar conditions. In addition, it is worth mentioning that stimulation above $10 \mathrm{~Hz}$ is likely to induce a prominent depression of GABAergic IPSCs, as reported in [36]. 
1 A well-tuned intensity can preferentially activate interneurons. Our results indicate that GABAergic

2 interneurons are recruited before pyramidal cells when a local bipolar stimulation at an accurately tuned intensity is used. The question is "why?".

4 A first hypothesis is cytoarchitectural. In cortical structures, interneurons are known to have extensive local axon ramifications as compared with the sparser branching of PC axons ([37-39], reviewed in [40]). In addition, through these highly divergent axons, inhibitory neurons contact most neighboring pyramidal cells $[41,42]$. Finally, based on combined electrical stimulation and twophoton calcium imaging, microstimulation sparsely activates axons in a volume tens of microns in diameter around the electrode even at low currents ([43], [44]). Therefore, the probability of eliciting action potentials in axons of interneurons is high upon direct stimulation. These action potentials synchronously triggered generate secondarily summed IPSPs in most surrounding PCs, as shown in the CA1 region of the hippocampus [45]. The subsequent LFP response is evocative of unitary inhibitory field potentials at monosynaptic latencies [45-47]. Indeed, these in vitro (hippocampal slices) studies showed that direct intracellular stimulation of single interneurons cells generate field potentials detected at multiple sites and over distances greater than $800 \mu \mathrm{m}$. The authors demonstrated that similar stimulation in single PCs is unable to generate a comparable field response. In addition, in the hippocampus, IPSPs have a larger amplitude than EPSPs [48]. To some extent, PSP-related parameters (rise time, decay time, average amplitude) in our neuronal population model are in agreement with these data as the inhibitory drive is much higher than the excitatory drive. Consequently, as shown in the results, direct stimulation of the inhibitory subpopulation favors the generation of a field response at weak stimulations intensities.

A second hypothesis relates to the resting membrane potential value of interneurons, which is 10 to $15 \mathrm{mV}$ higher (i.e. more depolarized) than that of PCs [49,50]. In addition, even if stimulation excites axons of PCs, the amplitude of EPSPs onto interneurons is two to four times greater than that 
generated onto PCs [24]. Due to these physiological properties, under weak electrical stimulation, interneurons will reach threshold faster and will generate APs before PCs.

Translation to patients. Reported results show the great potential of invasive electrical low intensity local bipolar stimulation for diagnosis particularly when hyperexcitability stems from a sustainable dysfunction of GABAergic processes that can be probed during interictal periods. It is noteworthy that overactivation of GABAergic interneurons can actively participate to initiating seizures [51]; but this effect might be due to excitatory GABAergic post-synaptic potentials [52][53]. These dynamic and rapid changes of GABAergic PSP effect during transition to seizure is not targeted by our method at the first intent.

In the patients considered in this study, intracerebral electrodes were used for both stimulation and recording of evoked LFP responses. NNEI values were found to be highly indicative of the excitability level in stimulated brain structures, as also assessed by inspection of intracerebral EEG signals showing either normal or locally-generated epileptiform activity. In patients with drug-resistant partial epilepsy who are candidate for surgery, intracerebral recording and stimulation is a routine procedure. In these patients, both healthy and epileptogenic regions are explored as part of the presurgical evaluation. In contrast to stimulation protocols aimed at modulating epileptiform discharges [54], the objective of our low-intensity ELBS is to locally probe the brain regions explored by depthelectrodes without changing the ongoing activity. The proposed method can potentially complement the standard explorations performed during pre-surgical evaluation of patients with drug-resistant epilepsy. Indeed, as based on an active paradigm (electrical stimulation), it can be used on all pairs of electrode contacts exploring brain regions in order to provide a quantified index characterizing underlying excitability. It does not require recording of interictal epileptiform events (which interpretation can be a matter of debate in some cases).

Today, the introduction of depth-electrodes in the human brain has been used in a large number of neurological disorders (Parkinson's disease, major depression, obsessive compulsive disorder, 
1 Alzheimer's disease, dystonia, addiction, chronic pain, ...) [55] offering the unique opportunity to

2 directly record from - and to probe - (dys)functioning neuronal networks.

\section{Supplementary Material}

4

\section{Supplementary Material 1 (SM1). Computation of the Neural Network Excitability Index (NNEI)}

The NNEI is derived from the Fourier Transform (FT) which provides both the amplitude and the phase of oscillatory components present in the signals. In practice, the NNEI computation involves three steps. First, the LFP signal recorded during periodic pulse stimulation is divided into single response epochs. Second, a Fast Fourier Transform (FFT) is performed on each epoch s yielding both the amplitude and phase of its frequency components, as complex-valued coefficients $Z_{f}^{s}$. Third, at each frequency $f$, the Phase Clustering Index (PCl, see [13] for details) is computed as the average of these complex coefficients, normalized by their magnitudes, over epochs:

$$
\operatorname{PCI}(f)=\left|\frac{\left.<Z_{f}^{s}\right\rangle_{s}}{\sqrt{\left\langle\left|Z_{f}^{s}\right|^{2}\right\rangle_{s}}}\right|
$$

$\operatorname{PCI}\left(f_{s}\right)$ is close to zero for randomly distributed phases, and close to 1 for coherent phases. Finally, in order to derive from $P C I\left(f_{s}\right)$ an index that is congruent with the excitability levels we define the NNEI given by $N N E I=1-P C\left(f_{s}\right)$ where $f_{s}$ denotes the stimulation frequency. The main difference between NNEI and the relative phase clustering index, or the $\mathrm{rPCl}$ introduced as $\max _{f}\left(P C I(f)-P C I\left(f_{s}\right)\right)$ in [56] is that while in the later the presence of high frequency harmonic components locked to the stimulus do contribute to the measurable effect, in the currently used NNEI quantity only the spectral components at the stimulation frequency are taken into account. The NNEI discloses low values, at low excitability levels, where LFP evoked responses (time locked to each pulse of the stimulation train) have similar time-courses, and high values, at high 
excitability levels, where LFPs have irregular time-courses, provided that the stimulation intensity is appropriately tuned.

Supplementary Material 2 (SM2). In vivo recordings, in vitro recordings and neuron labelling.

In vivo LFP recordings were performed in adult C57BL/6RJ male mice ( $80 \pm 5$ days). Surgery was performed under anesthesia (chloral hydrate $4 \%$ and xylazine $0.04 \%(10 \mu \mathrm{l} / \mathrm{g})$ in a stereotaxic frame $\left(\mathrm{KOPF}^{\circledR} 957\right)$ in a flat skull position. All mice were implanted with a bipolar depth electrode in the right hippocampus (CA1 region) and one monopolar surface electrode over the cerebellum (reference). Positions of hippocampi for implantation $(\mathrm{AP}=-2.0 \mathrm{~mm}, \mathrm{ML}=-1.5 \mathrm{~mm}$ or $\mathrm{ML}=+1.5 \mathrm{~mm}$, $\mathrm{DV}=-1.9 \mathrm{~mm}$ ) were chosen using mouse brain atlas [57]. Electrodes were made of a polyester insulated stainless steel wire $(\varnothing=139 \mu \mathrm{m})$ and fixed to the skull with cyanoacrylate and dental acrylic cement and finally connected to a female connector placed on top of the cement. Depth bipolar electrodes were assembled by twisting wires with a gap of approximately $0.5 \mathrm{~mm}$ between the higher and the lower contact. After surgery, animals were placed into individual cages where they could recover during three days (no EEG recording). During recordings, they were placed in a transparent Plexiglas cage, positioned in a Faraday cage. Animals were first habituated for one hour and then baseline data were recorded. At the end of in vivo recordings, brains were extracted and sliced (20 $\mu \mathrm{m}$ thickness) with a cryostat. All slices were colored using Cresyl violet acetate. Histological analyses were performed to verify the location of intracerebral electrodes.

In vitro recordings were performed in organotypic hippocampal slices as described in [23]. Slices were prepared from 6 day old Wistar rats and maintained for 3-6 weeks in vitro using the roller drum technique. Animals were killed by decapitation. Neurons were visualized with DIC optics. Signals were amplified with an Axopatch 200B amplifier (Axon Instruments, Foster City, CA, USA), filtered at $2 \mathrm{kHz}$, stored and analyzed with pClamp9 software (Axon Instruments). All animal procedures were performed in accordance with Swiss law, with strict attention given to the care and use of animals. 
1 The protocols for our experiments were approved by the Ethics Committee of the Veterinary

2 Department of the Canton of Zurich (Approval ID 41/2011).

Drugs. Concentrated stock solutions of drugs were prepared in distilled water or dimethylsulfoxide (not exceeding a final concentration of $0.02 \%$ ), stored at $-20^{\circ} \mathrm{C}$ in single-use aliquots, thawed and diluted in ACSF immediately before use. Bicuculline was purchased from Sigma, and tetrodotoxin (TTX), NBQX (2,3-Dioxo-6-nitro-1,2,3,4-tetrahydrobenzo[f]quinoxaline-7-sulfonamide), from Abcam Biochemicals (Cambridge, UK).

Pyramidal cells and Interneurons. Intracellular CA1 pyramidal cell recordings were made with the patch-clamp technique in whole-cell current-clamp mode in stratum pyramidale, and were identified according to their electrophysiological properties, their morphology and the presence of dendritic spines. Interneurons were identified by their firing properties (low adaptation), presence of a large $\mathrm{I}_{\text {AHP, }}$, their morphology and an absence of dendritic spines (as in (Gee et al., 2010)) .

Extracellular local bipolar stimulation. Parallel bipolar electrodes (FHC Corporate and Manufacturing, PB SA 1075, Bowdoin, USA) were used for local stimulation. Space between the two electrodes was manually reduced to $500 \mu \mathrm{m}$. Stimulation was applied across CA1 pyramidal layer (one electrode in $S$. Oriens and the other in S. Radiatum of the CA1 subfield) unless specified.

Neuron Labelling. After recording, slices were fixed $4-12 \mathrm{~h}$ in $4 \%$ paraformaldehyde in $0.1 \mathrm{M}$ phosphate buffer $\mathrm{pH}$ 7.4. Slices were washed in $0.1 \mathrm{M}$ phosphate buffer then removed from the coverslips, washed in $0.1 \mathrm{M}$ phosphate buffer and permeabilized in $0.1 \mathrm{M}$ phosphate buffer, $0.4 \%$ Triton X-100 and 5\% heat-inactivated horse serum for $24 \mathrm{~h}$ at $4{ }^{\circ} \mathrm{C}$. Slices were then processed with streptavidin-conjugated AlexaFluor-488 (4 $\mathrm{gg} / \mathrm{ml}$, Invitrogen) to reveal biocytin-filled neurons. To visualize the layers of the hippocampus, a primary antibody that recognizes mature neurons (neuronal nuclear antigen, mouse anti-NeuN 1:500, Chemicon, Temecula, CA, USA) was incubated for $24 \mathrm{~h}$ at $4^{\circ} \mathrm{C}$ in $0.1 \mathrm{M}$ phosphate-buffered saline with $0.4 \%$ Triton $\mathrm{X}-100$ and $3 \%$ heat-inactivated 
1

2

3

4

5

6

7

8

9

$18 \sim \mathcal{N}\left(\sum_{i=1}^{N} m_{\gamma^{i}}, \sum_{i=1}^{N} \sigma_{\gamma^{i}}^{2}\right)$. Thus, the acceptance region at the (1-p) level of confidence for the hypothesis normal horse serum (NHS). Cultures were washed with 0.1 M PB 0.4\% Triton X-100 (4-30 min) and incubated at $4^{\circ} \mathrm{C}$ overnight with donkey anti-mouse Alexa 546 (1:1000, Molecular Probes, Eugene, OR, USA) in $0.1 \mathrm{M} \mathrm{PB}, 0.4 \%$ Triton $\mathrm{X}-100$ and 3\% heat-inactivated horse serum. After extensive washings in 0.1M PB sections were mounted onto gelatin-coated slides with Vectashield-mounting medium (Reactolab) to preserve fluorescent labeling and stored in the dark at $4{ }^{\circ} \mathrm{C}$. Image stacks were collected with a laser scanning confocal microscope (Olympus IX 81, Olympus, Tokyo, Japan) equipped with a $40 x$ objective (N.A. 1.25).

\section{Supplementary Material 3 (SM3). Statistical test for optimal stimulation intensity}

\section{The optimal intensity value corresponds to the intensity for which the cross-correlation among} stimulation evoked LFP responses becomes significant, and thus the null-hypothesis, $\left(\mathrm{H}_{0}: \rho_{x y}^{i}=0\right)$, can be rejected. The proposed test starts with the computation of the value $r_{x y}^{i}$ of the linear correlation coefficient $\rho_{x y}^{i}$ for any pair $i$ of evoked responses $X$ and $Y$. A log-transform of $r_{x y}^{i}$ is introduced: $\gamma^{i}=\frac{1}{2} \ln \left[\left(1+r_{x y}^{i}\right) /\left(1-r_{x y}^{i}\right)\right]$. It can be shown that the obtained random variable $\gamma^{i}$ is normally distributed with mean $m_{\gamma^{i}}=\frac{1}{2} \ln \left[\left(1+\rho_{x y}^{i}\right) /\left(1-\rho_{x y}^{i}\right)\right]$ and variance $\sigma_{\gamma^{i}}^{2}=1 /(m-3)$ under the null hypothesis $\mathrm{H}_{0}: \rho_{x y}^{i}=0$, where $m$ is the size of evoked LFP responses (\# samples).

The sum $\Gamma$ of $\gamma^{i}$ values over the $N=n(n-1) / 2$ pairs of evoked responses (for simplicity considered as independent) recorded from a stimulation train of $n$ pulses is also Gaussian: $\Gamma$ 19 of absence of correlation among evoked responses is given by $20-z_{p / 2} \leq w=\frac{\sqrt{m-3}}{2 N} \sum_{i=1}^{N} \ln \left[\frac{1+r_{x y}^{i}}{1-r_{x y}^{i}}\right] \leq z_{p / 2}$, where $\mathrm{z}$ is the normalized variable. It follows that values 
1 outside the interval $[-2.576,+2.576]$ constitute evidence for the existence of a correlation between

2 evoked responses at $99 \%$ level of confidence ( $p$-value $\left.=0.01, z_{0.005}=-2.576\right)$. Supplementary Fig. S1

3 shows the evolution of the NNEI computed from simulated LFPs, for three excitability states (low,

4 med and high) and for three stimulation intensity values (optimal, optimal divided by 3 and optimal

5 times 3 ). The optimal I-value (1.5 a.u) was that corresponding to $w=2.6$ (i.e. just above statistical

6 threshold 2.576). As depicted, for this precise intensity, the NNEI can effectively discriminate the

7 three excitability states. In contrast, when the intensity is too low $(w<<2.576)$ or two high ( $w$

$8 \quad$ > 2.576), NNEI values are not indicative of the excitability level in the model.

\section{Acknowledgements}

This work was supported by "Region Bretagne” (« EPIGONE » project, CREATE Competitive Call) and by the Swiss National Science Foundation (31-45547.95). Authors wish to thank Dr. Julien Modolo for

12 helpful comments on this MS. 


\section{References}

1. Xue M, Atallah BV, Scanziani M (2014) Equalizing excitation-inhibition ratios across visual cortical neurons. Nature 511: 596-600.

2. Southwell DG, Nicholas CR, Basbaum Al, Stryker MP, Kriegstein AR, et al. (2014) Interneurons from Embryonic Development to Cell-Based Therapy. Science 344: 1240622-1240622.

3. Olmos-Serrano JL, Paluszkiewicz SM, Martin BS, Kaufmann WE, Corbin JG, et al. (2010) Defective GABAergic Neurotransmission and Pharmacological Rescue of Neuronal Hyperexcitability in the Amygdala in a Mouse Model of Fragile X Syndrome. Journal of Neuroscience 30: 99299938.

4. Han S, Tai C, Westenbroek RE, Yu FH, Cheah CS, et al. (2012) Autistic-like behaviour in Scn1a+/mice and rescue by enhanced GABA-mediated neurotransmission. Nature 489: 385-390.

5. Pérez-Cremades D, Hernández S, Blasco-Ibáñez JM, Crespo C, Nacher J, et al. (2010) Alteration of inhibitory circuits in the somatosensory cortex of Ts65Dn mice, a model for Down's syndrome. Journal of Neural Transmission 117: 445-455.

6. Lin L-C, Sibille E (2013) Reduced brain somatostatin in mood disorders: a common pathophysiological substrate and drug target? Frontiers in Pharmacology 4.

7. Zeilhofer HU, Wildner H, Yevenes GE (2012) Fast Synaptic Inhibition in Spinal Sensory Processing and Pain Control. Physiological Reviews 92: 193-235.

8. Ben-Ari Y (2006) Seizures beget seizures: the quest for GABA as a key player. Crit Rev Neurobiol 18: 135-144.

9. Mclntyre CC, Chaturvedi A, Shamir RR, Lempka SF (2014) Engineering the Next Generation of Clinical Deep Brain Stimulation Technology. Brain Stimul.

10. Fisher RS, Velasco AL (2014) Electrical brain stimulation for epilepsy. Nature Reviews Neurology 10: 261-270.

11. Parra J, Kalitzin SN, Iriarte J, Blanes W, Velis DN, et al. (2003) Gamma-band phase clustering and photosensitivity: is there an underlying mechanism common to photosensitive epilepsy and visual perception? Brain 126: 1164-1172.

12. Kalitzin S, Velis D, Suffczynski P, Parra J, da Silva FL (2005) Electrical brain-stimulation paradigm for estimating the seizure onset site and the time to ictal transition in temporal lobe epilepsy. Clin Neurophysiol 116: 718-728.

13. Suffczynski P, Kalitzin S, da Silva FL, Parra J, Velis D, et al. (2008) Active paradigms of seizure anticipation: computer model evidence for necessity of stimulation. Phys Rev E Stat Nonlin Soft Matter Phys 78: 051917.

14. Wendling F, Benquet P, Bartolomei F, Jirsa V (2016) Computational models of epileptiform activity. J Neurosci Methods 260: 233-251.

15. Wendling F, Bartolomei F, Mina F, Huneau C, Benquet P (2012) Interictal spikes, fast ripples and seizures in partial epilepsies--combining multi-level computational models with experimental data. Eur J Neurosci 36: 2164-2177.

16. Kepecs A, Fishell G (2014) Interneuron cell types are fit to function. Nature 505: 318-326.

17. Jansen BH, Zouridakis G, Brandt ME (1993) A neurophysiologically-based mathematical model of flash visual evoked potentials. Biol Cybern 68: 275-283.

18. Wendling F, Bartolomei F, Bellanger JJ, Chauvel P (2002) Epileptic fast activity can be explained by a model of impaired GABAergic dendritic inhibition. Eur J Neurosci 15: 1499-1508.

19. Wendling F, Hernandez A, Bellanger JJ, Chauvel P, Bartolomei F (2005) Interictal to ictal transition in human temporal lobe epilepsy: insights from a computational model of intracerebral EEG. J Clin Neurophysiol 22: 343-356.

20. Wendling F, Bellanger JJ, Bartolomei F, Chauvel P (2000) Relevance of nonlinear lumpedparameter models in the analysis of depth-EEG epileptic signals. Biol Cybern 83: 367-378.

21. Molaee-Ardekani B, Marquez-Ruiz J, Merlet I, Leal-Campanario R, Gruart A, et al. (2013) Effects of transcranial Direct Current Stimulation (tDCS) on cortical activity: a computational modeling study. Brain Stimul 6: 25-39. 
22. McIntyre CC, Grill WM (2002) Extracellular stimulation of central neurons: influence of stimulus waveform and frequency on neuronal output. J Neurophysiol 88: 1592-1604.

23. Gahwiler BH, Capogna M, Debanne D, McKinney RA, Thompson SM (1997) Organotypic slice cultures: a technique has come of age. Trends Neurosci 20: 471-477.

24. Debanne D, Guerineau NC, Gahwiler BH, Thompson SM (1995) Physiology and pharmacology of unitary synaptic connections between pairs of cells in areas CA3 and CA1 of rat hippocampal slice cultures. J Neurophysiol 73: 1282-1294.

25. Barba C, Barbati G, Minotti L, Hoffmann D, Kahane P (2007) Ictal clinical and scalp-EEG findings differentiating temporal lobe epilepsies from temporal 'plus' epilepsies. Brain 130: 19571967.

26. Mandhane SN, Aavula K, Rajamannar T (2007) Timed pentylenetetrazol infusion test: a comparative analysis with s.c.PTZ and MES models of anticonvulsant screening in mice. Seizure 16: 636-644.

27. Pitkanen A, Kharatishvili I, Karhunen H, Lukasiuk K, Immonen R, et al. (2007) Epileptogenesis in experimental models. Epilepsia 48 Suppl 2: 13-20.

28. Schwartzkroin PA (1994) Cellular electrophysiology of human epilepsy. Epilepsy Res 17: 185-192.

29. Kato M, Dobyns WB (2005) X-linked lissencephaly with abnormal genitalia as a tangential migration disorder causing intractable epilepsy: proposal for a new term, "interneuronopathy". Journal of Child Neurology 20: 392-397.

30. Price MG, Yoo JW, Burgess DL, Deng F, Hrachovy RA, et al. (2009) A triplet repeat expansion genetic mouse model of infantile spasms syndrome, $\operatorname{Arx}(\mathrm{GCG}) 10+7$, with interneuronopathy, spasms in infancy, persistent seizures, and adult cognitive and behavioral impairment. The Journal of Neuroscience: The Official Journal of the Society for Neuroscience 29: 8752-8763.

31. Loup F, Wieser HG, Yonekawa Y, Aguzzi A, Fritschy JM (2000) Selective alterations in GABAA receptor subtypes in human temporal lobe epilepsy. J Neurosci 20: 5401-5419.

32. Cossart R, Dinocourt C, Hirsch JC, Merchan-Perez A, De Felipe J, et al. (2001) Dendritic but not somatic GABAergic inhibition is decreased in experimental epilepsy. Nat Neurosci 4: 52-62.

33. Mormann F, Andrzejak RG, Elger CE, Lehnertz K (2007) Seizure prediction: the long and winding road. Brain 130: 314-333.

34. McIntyre CC, Grill WM (1999) Excitation of central nervous system neurons by nonuniform electric fields. Biophys J 76: 878-888.

35. McIntyre CC, Grill WM (2001) Finite element analysis of the current-density and electric field generated by metal microelectrodes. Ann Biomed Eng 29: 227-235.

36. Gonzalez-Burgos G, Krimer LS, Povysheva NV, Barrionuevo G, Lewis DA (2005) Functional properties of fast spiking interneurons and their synaptic connections with pyramidal cells in primate dorsolateral prefrontal cortex. J Neurophysiol 93: 942-953.

37. Freund TF, Buzsaki G (1996) Interneurons of the hippocampus. Hippocampus 6: 347-470.

38. Vida I, Halasy K, Szinyei C, Somogyi P, Buhl EH (1998) Unitary IPSPs evoked by interneurons at the stratum radiatum-stratum lacunosum-moleculare border in the CA1 area of the rat hippocampus in vitro. J Physiol 506 ( Pt 3): 755-773.

39. Martina $M$, Vida I, Jonas $P$ (2000) Distal initiation and active propagation of action potentials in interneuron dendrites. Science 287: 295-300.

40. Markram H, Toledo-Rodriguez M, Wang Y, Gupta A, Silberberg G, et al. (2004) Interneurons of the neocortical inhibitory system. Nat Rev Neurosci 5: 793-807.

41. Fino E, Yuste R (2011) Dense inhibitory connectivity in neocortex. Neuron 69: 1188-1203.

42. Packer AM, Yuste R (2011) Dense, unspecific connectivity of neocortical parvalbumin-positive interneurons: a canonical microcircuit for inhibition? J Neurosci 31: 13260-13271.

43. Histed MH, Bonin V, Reid RC (2009) Direct activation of sparse, distributed populations of cortical neurons by electrical microstimulation. Neuron 63: 508-522.

44. Borchers S, Himmelbach M, Logothetis N, Karnath HO (2012) Direct electrical stimulation of human cortex - the gold standard for mapping brain functions? Nat Rev Neurosci 13: 63-70. 
45. Ali AB, Bannister AP, Thomson AM (1999) IPSPs elicited in CA1 pyramidal cells by putative basket cells in slices of adult rat hippocampus. Eur J Neurosci 11: 1741-1753.

46. Glickfeld LL, Roberts JD, Somogyi P, Scanziani M (2009) Interneurons hyperpolarize pyramidal cells along their entire somatodendritic axis. Nat Neurosci 12: 21-23.

47. Bazelot M, Dinocourt C, Cohen I, Miles R (2010) Unitary inhibitory field potentials in the CA3 region of rat hippocampus. J Physiol 588: 2077-2090.

48. Biro AA, Holderith NB, Nusser Z (2006) Release probability-dependent scaling of the postsynaptic responses at single hippocampal GABAergic synapses. J Neurosci 26: 12487-12496.

49. Jonas P, Bischofberger J, Fricker D, Miles R (2004) Interneuron Diversity series: Fast in, fast out-temporal and spatial signal processing in hippocampal interneurons. Trends Neurosci 27: 3040.

50. Campanac E, Gasselin C, Baude A, Rama S, Ankri N, et al. (2013) Enhanced intrinsic excitability in basket cells maintains excitatory-inhibitory balance in hippocampal circuits. Neuron 77: 712722.

51. de Curtis M, Avoli M (2016) GABAergic networks jump-start focal seizures. Epilepsia.

52. Shiri Z, Manseau F, Levesque M, Williams S, Avoli M (2015) Interneuron activity leads to initiation of low-voltage fast-onset seizures. Ann Neurol 77: 541-546.

53. Zhang ZJ, Koifman J, Shin DS, Ye H, Florez CM, et al. (2012) Transition to seizure: ictal discharge is preceded by exhausted presynaptic GABA release in the hippocampal CA3 region. J Neurosci 32: 2499-2512.

54. Koubeissi MZ, Kahriman E, Syed TU, Miller J, Durand DM (2013) Low-frequency electrical stimulation of a fiber tract in temporal lobe epilepsy. Ann Neurol 74: 223-231.

55. Lozano AM, Lipsman N (2013) Probing and regulating dysfunctional circuits using deep brain stimulation. Neuron 77: 406-424.

56. Kalitzin S, Parra J, Velis DN, Lopes da Silva FH (2002) Enhancement of phase clustering in the EEG/MEG gamma frequency band anticipates transitions to paroxysmal epileptiform activity in epileptic patients with known visual sensitivity. IEEE Trans Biomed Eng 49: 1279-1286.

57. Paxinos G, Franklin KBJ (2001) The Mouse Brain in Stereotaxic Coordinates: Academic Press. 


\section{1 \\ Legends}

2

Table 1: Clinical data for the four patients (P1 - P4) who underwent ELBS. Clinical results are shown in Figures 6 and 7.

Table 2: Technical features of electrodes used for in vivo, in vitro and clinical measurements.

Figure 1: Computational modeling approach carried out to analyze ELBS effects on a population of neurons.

A: LFP signals in response to ELBS were simulated from a NMM representing a local population of neurons. In this model, the excitability level could be tuned and the Neural Network Excitability Index could be computed from LFP signals. From with comparison, a number of predictions were made regarding ELBS.

B: The neuronal population model used to simulate LFPs under pulse stimulation comprises 2 subpopulations of neurons (PCs and INs). Stimulation can be applied on PCs and/or INs.

C: Computational modeling of local field potentials (LFPs) under pulse stimulation. Block diagram of the model showing the positive (excitatory) and negative (inhibitory) feedback loops in which the linear transfer function $h$ and the nonlinear function $S$ account for the relationship between the two main variables of the model, namely the membrane potential and the firing rate at each subpopulation. Pulse stimulation effects were modeled as a perturbation of the mean membrane potential in PC and IN subsets, that linearly depends on the externally-applied current intensity.

Figure 2: Predictions from a computational model on the impact of low intensity local stimulation on a neuronal population.

A: Spontaneous activity. LFP signals simulated with the computational model for parameters mimicking low/high levels of excitability (by increasing the ratio between EPSP/IPSP amplitudes): the spontaneously-generated background activity did not reveal noticeable differences in time-series and power spectra.

B: Stimulation-based paradigm. Top: Result of electric stimulation (pulses at $8 \mathrm{~Hz}$, low intensity direct stimulation) applied both to pyramidal cells and interneurons (arrows) under low/high levels of excitability. Bottom: superimposed LFP raw traces (time-locked to the stimuli). Note that for the high excitability condition, superimposed responses have an irregular time course. Right: Averages of Neural Network Excitability Index (NNEI) values according to low, medium and high (Lo, Me and Hi) f excitability levels. 
1 C: Left: Electric stimulation applied only to IN population: LFP responses and NNEI index for three 2 excitability levels (Lo, Me, Hi). Right: Idem but electric stimulation applied only to PC population. The NNEI was able to distinguish between the three simulated excitability conditions (Lo, Med, $\mathrm{Hi}$ ) in the former (left) but not in the latter (right) case.

D: Impact of the stimulation intensity (I-value) on simulated responses for the three excitability conditions. Left: superimposed traces obtained for very low, intermediate and high I-values. Right: for a very low I-value (dark to light blue color) and a high I-value (red to orange color), the NNEI was either - blind to - or - not fully informative of - the excitability condition. In contrast, for intermediate I-values (yellow to blue color), the NNEl showed conspicuous differences with respect to excitability levels.

E: Effects of the stimulation frequency. Periodic stimulation was applied in the model for various excitability conditions (Lo, Me and $\mathrm{Hi}$ ). The stimulation frequency varied from 1 to $40 \mathrm{~Hz}$.

F: For a stimulation frequency ranging from 1 to $10 \mathrm{~Hz}$ (blue, green curves), the NNEI computed for simulated signals is indicative of the underlying excitability state. Beyond $10 \mathrm{~Hz}$ (orange to red), the contrast between low and high NNEI values decreases and leads to equivocal results.

\section{Figure 3: Calibration and evaluation of the NNEI in vivo.}

Calibration of the stimulation intensity both in silico and in vivo (hippocampus, mouse). LFPs elicited by pulse train stimulation $(8 \mathrm{~Hz})$ in the computational model ( $\mathbf{A}$ - left) and in vivo (B - right). Note that a small response is visible in simulated and real LFPs after each stimulation pulse. A LFP undistinguishable from noise was obtained for a low intensity (1-3 a.u, in silico) and (.6 - $1.0 \mu \mathrm{A}$ in vivo). A small deflection was visible for intermediate stimulation intensity (3-4.5 a.u in silico; 1.0-1.8 $\mu \mathrm{A}$ in vivo). A prominent wave appeared for high stimulation value ( $>4.5$ a.u in silico; $>1.8 \mu \mathrm{A}$ in vivo). The optimal stimulation intensity can be obtained using a statistical test (mean w above threshold) of the linear correlation among evoked responses (see methods). In practice, the mean $w$ test confirmed the visual inspection of the LFP to determine the optimal intensity. Histograms showing a similar evolution of NNEI values as a function of the stimulation intensity in the computational model and in mice.

C. Typical LFP recordings performed in vivo before and after PTZ injection. Left: pulse train stimulation. Middle: magnified evoked LFP response. Note the presence of the small deflection subsequent to each pulse, before PTZ injection. Note that this deflection disappears 10 min after PTZ injection. Right: superimposed evoked LFP responses in both conditions. Note the irregular, variable time course of responses after PTZ injection. Similar results were obtain in 6 mice.

D. Evolution of average (PTZ: $n=6$, control: $n=6$ ) NNEI values before and after injection. Right: comparison between max. NNEI values computed before and after injection (from $t=5$ to $t=10 \mathrm{~min}$ ). 
2 Figure 4: ELBS-induced suprathreshold LFP response and hyperpolarization of pyramidal cells in organotypic slice culture.

A: Simultaneous field (stratum radiatum) and patch-clamp recording (current clamp, CA1 pyramidal cell layer) performed during extracellular local bipolar stimulation - ELBS - (bipolar electrodes placed in CA1 area, in S-Oriens and S-Radiatum). Pyramidal cells (PC) were characterized by their morphology, presence of dendritic spines and firing pattern. Note the low frequency of the firing pattern and the attenuation upon current clamp depolarization.

B: Progressive increase of ELBS intensity $(70,100,170,230 \mu \mathrm{A})$ during simultaneous recording of intracellular membrane potential of a single CA1 PC (patch-clamp, current-clamp mode) and extracellular field recording (LFP). Note that a visible deflection appeared in the LFP simultaneously with PC hyperpolarization.

C: Example of reproducible LFP and PC hyperpolarization evoked by optimal ELBS stimulation under low excitability condition (ACSF). D: Increasing excitability of organotypic slice by application of low$\mathrm{Mg}^{2+}$ modified ACSF + bicuculline induced LFP response variability and corresponding increase of the NNEI. Example of LFP during superfusion of normal ACSF and following bath application of low- $\mathrm{Mg}^{2+}$ modified ACSF + bicuculline $(10 \mu \mathrm{M})$. Note that under the high excitability condition superimposed LFP responses were less coherent. Histogram: significantly different average NNEI values were obtained for low (ACSF) or high excitability condition (low-Mg ${ }^{2+}+$ bicuculline), as calculated from LFPs $(\mathrm{n}=5, \mathrm{p}<0.001)$, in vitro.

\section{Figure 5: ELBS induces GABAergic IPSPs in pyramidal cells.}

A: ELBS induced hyperpolarization was fully and reversibly blocked by the $G A B A_{A}$ receptor antagonist bicuculline $(10 \mu \mathrm{M})$ and by the voltage-gated sodium channel blocker TTX $(1 \mu \mathrm{M})$. Illustrative traces are shown below. Similar results were obtain on 8 (Bic) and 6 (TTX) cells.

B: Pyramidal cells were depolarized in current clamp mode. ELBS induced a hyperpolarization and transiently stopped the firing of PCs.

C: Progressively increasing ELBS intensity $(50,100,150,250 \mu \mathrm{A})$ induced suprathreshold IPSPs (100 $150 \mu \mathrm{A})$. Stronger stimulation $(250 \mu \mathrm{A})$ induced first an EPSP.

D: Average amplitude of CA1 postsynaptic potentials measured after ELBS (CA1 area local stimulation and CA1 PC recording, left) relative to stimulation intensity. Suprathreshold evoked postsynaptic potentials were inhibitory (negative value) for local stimulation..

E: Histogram showing the percentage of pyramidal cells responding with an IPSP or an EPSP after optimal ELBS, high intensity ELBS, or remote extracellular stimulation (CA3 area). Average results shown in C, D, E were obtained from 32 cells. 
2 Figure 6: Clinical investigation (Patient P1, detailed).

3 A: MRI data from a patient with temporal lobe epilepsy, prior to surgery. This patient underwent intracerebral EEG exploration (iEEG, stereo-electro-encephalographic technique) as part of the presurgical assessment. For simplicity, only the two depth-electrodes positioned in the left (LHIP, red color) and right (RHIP, blue color) hippocampus are indicated in the MRI coronal view. Two contacts were chosen to perform ELBS $(0.5 \mathrm{~mA}, 10 \mathrm{~Hz}$, biphasic pulses, $100 \mu \mathrm{s} /$ phase, 6 trains of $5 \mathrm{sec})$.

B: iEEG signals simultaneously recorded from the two contacts located in both hippocampi during a sub-clinical seizure (no symptoms). Epileptiform activity only involved the LHIP. Meanwhile, no visible change could be detected in the background activity recorded from the RHIP.

C: iEEG signals recorded during ELBS from both hippocampi (non-simultaneously). Outside stimulation pulse trains, the activity does not change significantly. Several interictal epileptic spikes can be observed in the LHIP.

D: Raw stimulation-induced evoked responses time-locked to the stimulation onset (black lines) and averaged over responses (blue and red lines). On the left side (LHIP), the time-course of responses appears as more irregular when compared with the right side (RHIP). The negative deflection is more pronounced on the average response of the RHIP as compared with LHIP.

E: NNEI values computed from the left (LHIP) and the right (RHIP) hippocampi for the 6 trains of 50 pulses. NNEI values are relatively stable over time. NNEI values recorded from LHIP are significantly higher than those recorded from RHIP (Mann Whitney U-test, $p<0.01$ ).

Figure 7: Clinical investigation (Patients P2, P3 and P4).

A, B: In patients P2 and P3, high NNEI values $(0.3<$ NNEI $<0.5)$ were computed from evoked responses in SEEG signals recorded in both right and left hippocampi (RHIP, LHIP). This abnormal excitability is confirmed by the presence of locally-generated epileptic spikes that occur either asynchronously or simultaneously at both sides (right panels).

C: In patient P4, NNEI values were computed from SEEG signals recorded in the right hippocampus (RHIP) and in the right middle temporal gyrus (RMTG). The strong contrast (RHIP: NNEI=0.34, RMTG: NNEI=0.04) suggests abnormal excitability in the RHIP and healthy tissue in the RMTG. SEEG signals show rhythmic discharges of epileptic spikes occurring in the RHIP with simultaneous normal activity in the RMTG (right panel). 

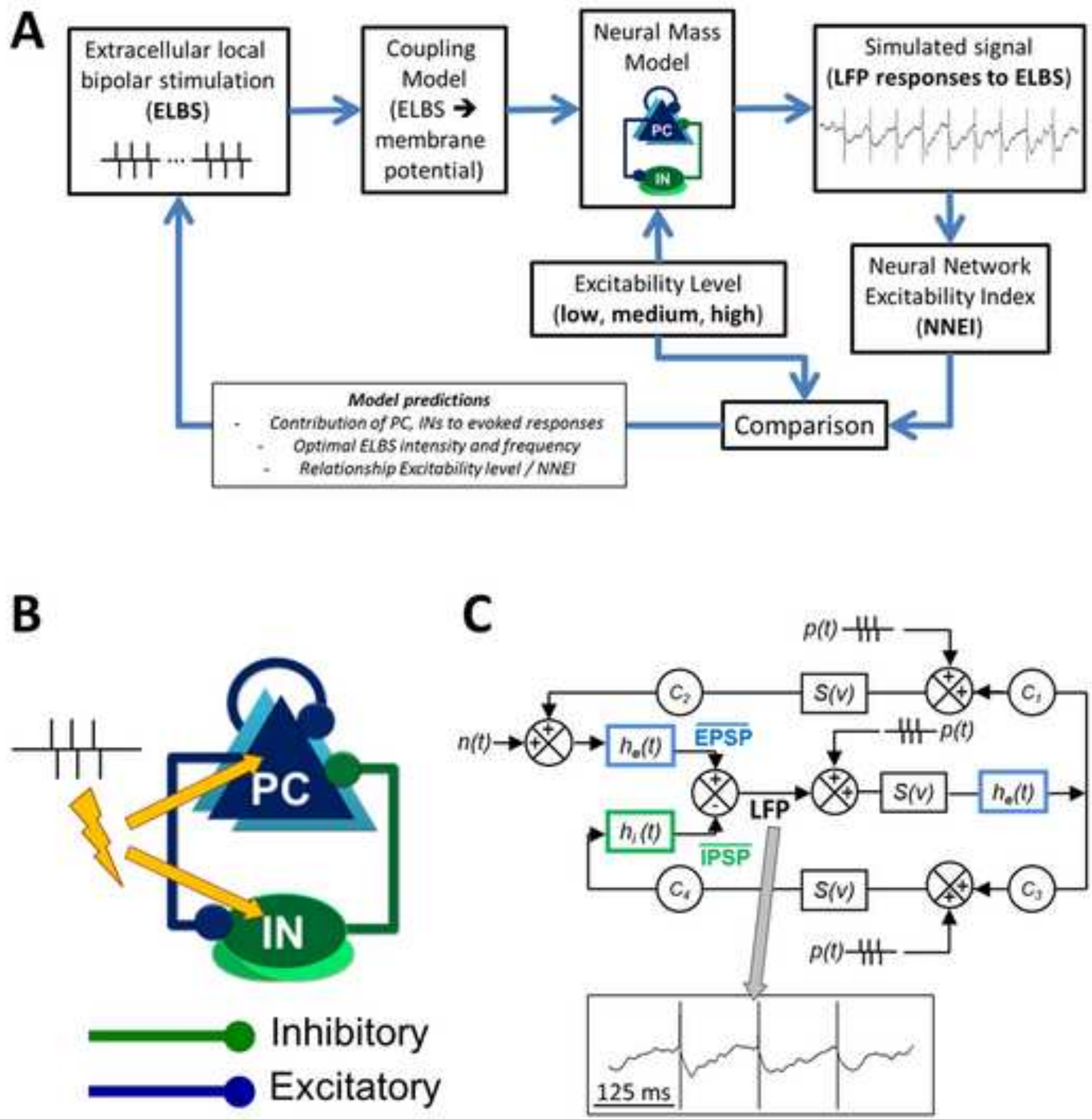
Figure 2
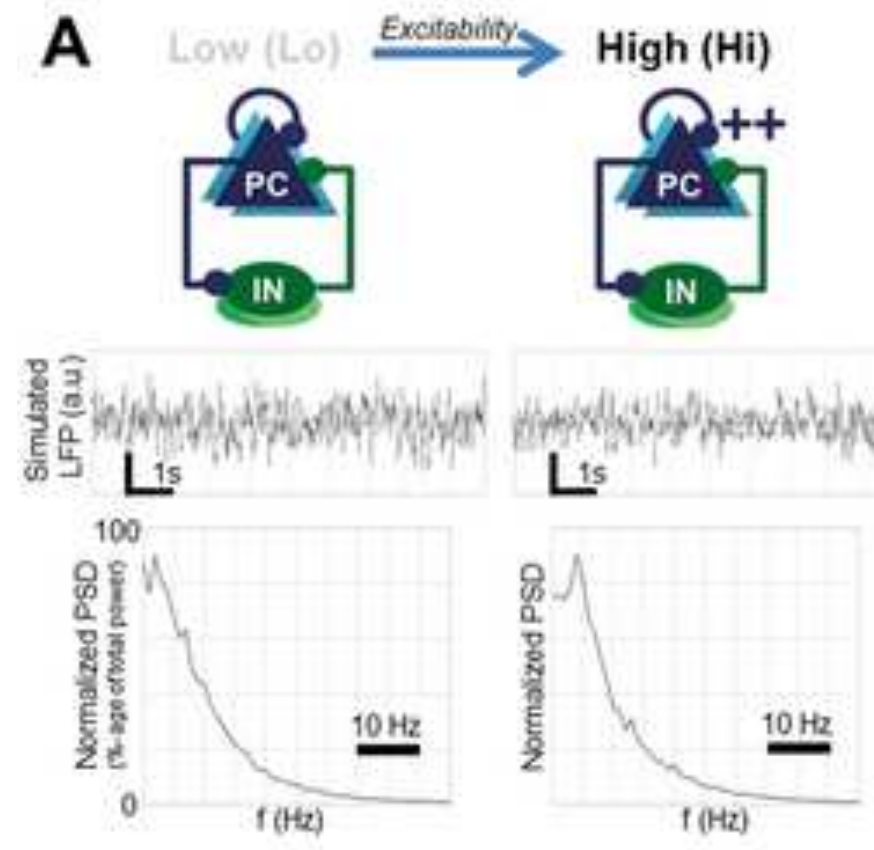

B Low (Lo) $\stackrel{\text { Excitabinity }}{\longrightarrow}$ High (Hi)
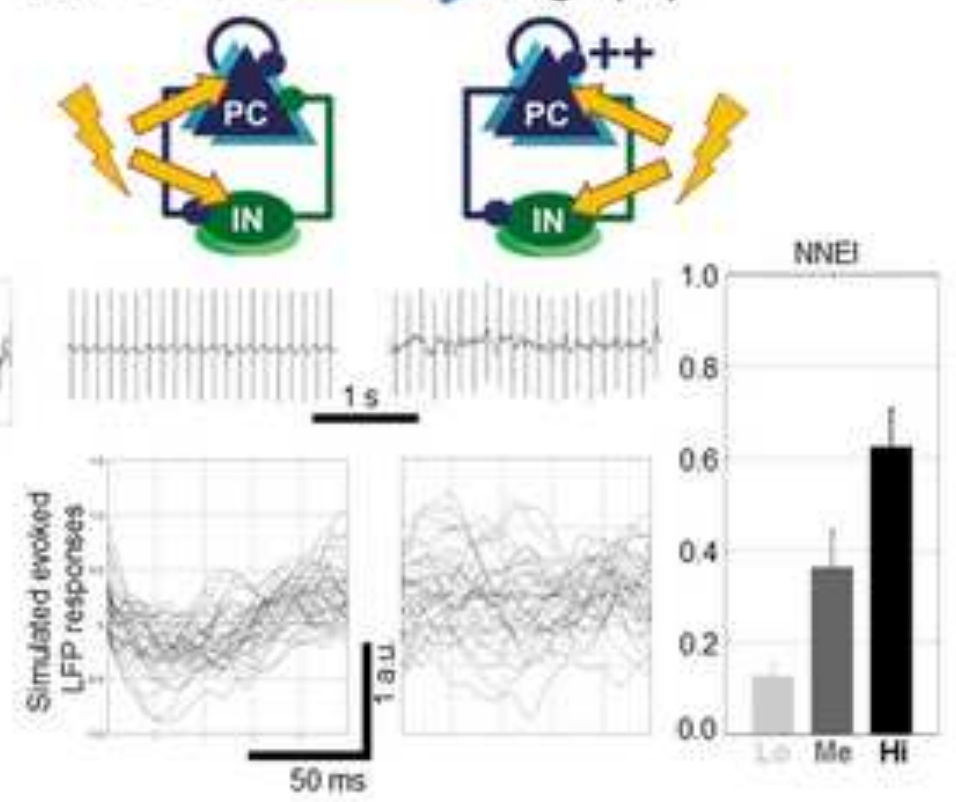

C
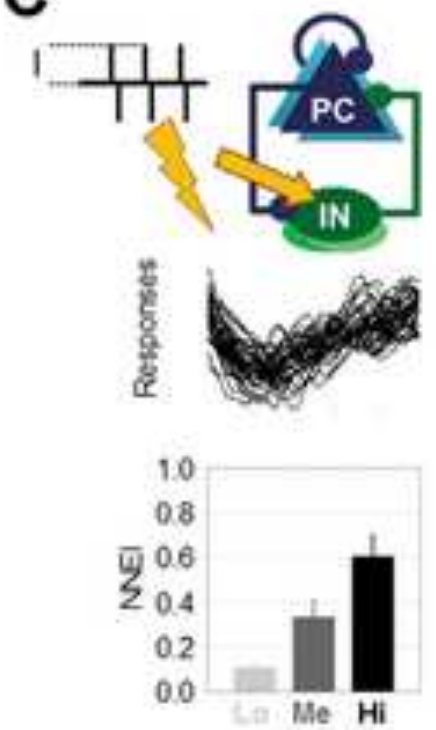

E
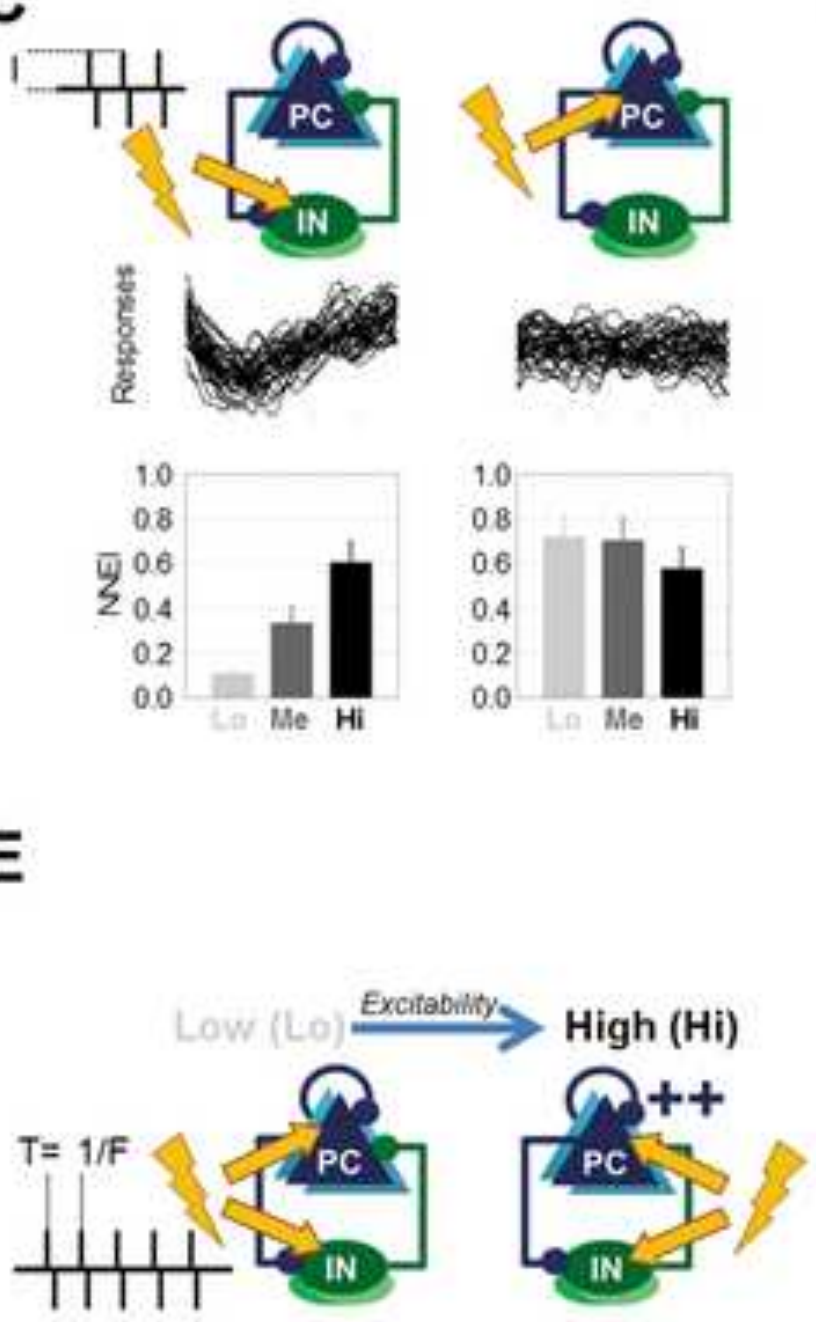

D

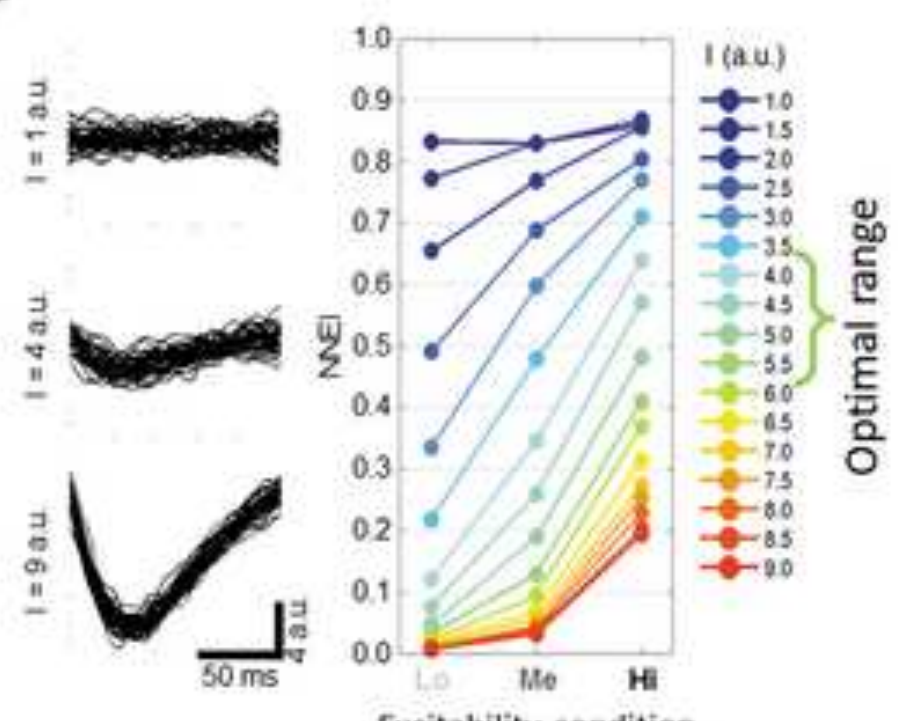

Excitability condition
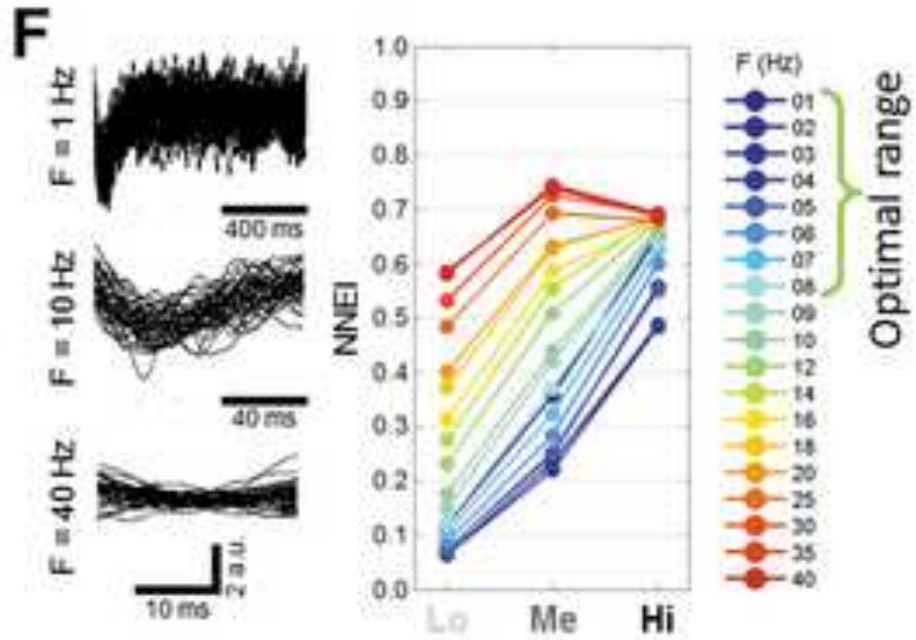

Excitability condition 
A

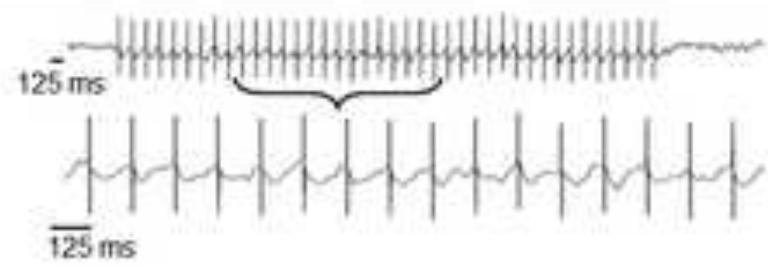

Superimposed rosponses

I-value: too low optimal
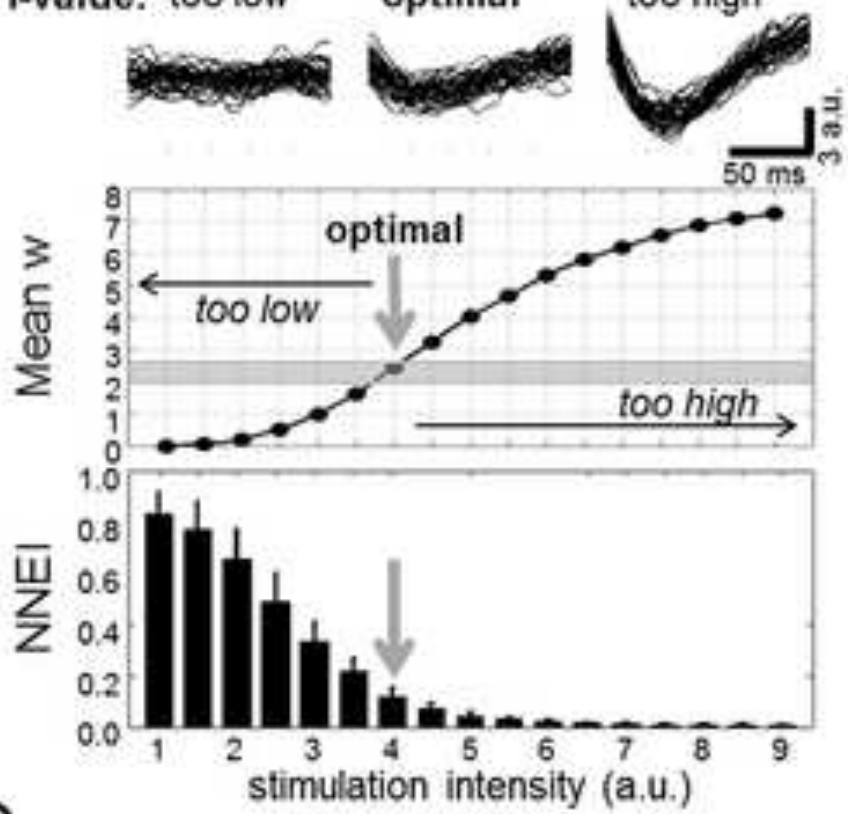

C
B

In vivo

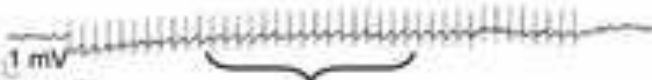

$125 \mathrm{~ms}$

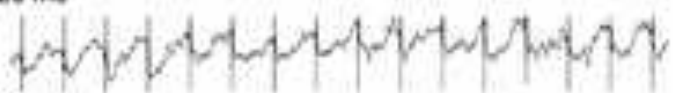
$200 \mathrm{WV}$

$125 \mathrm{~ms}$

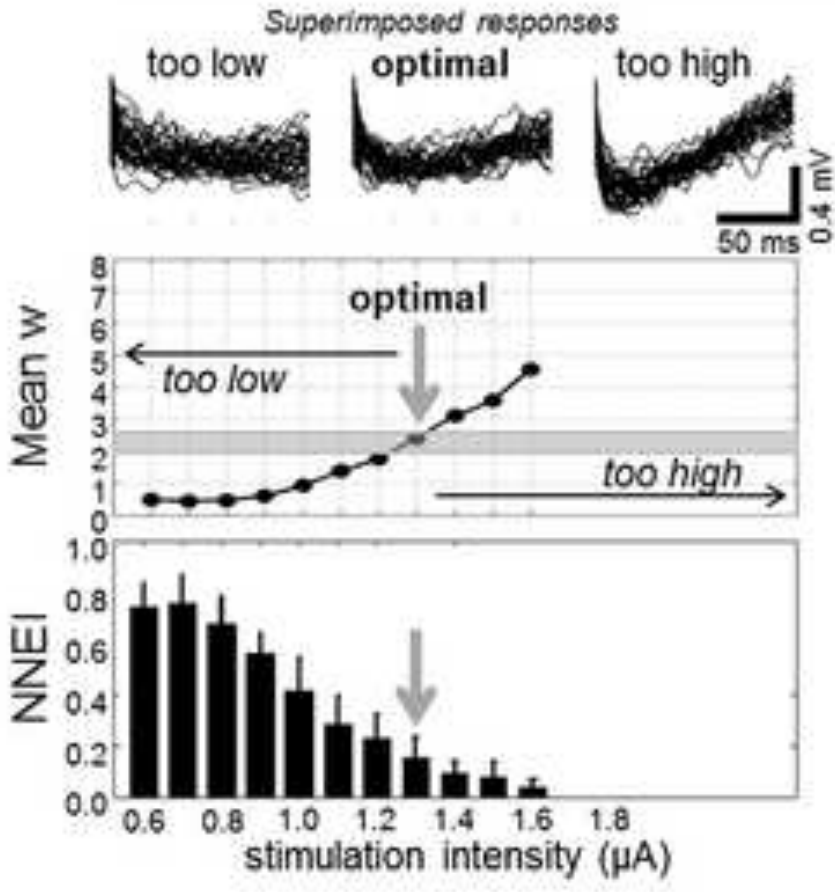

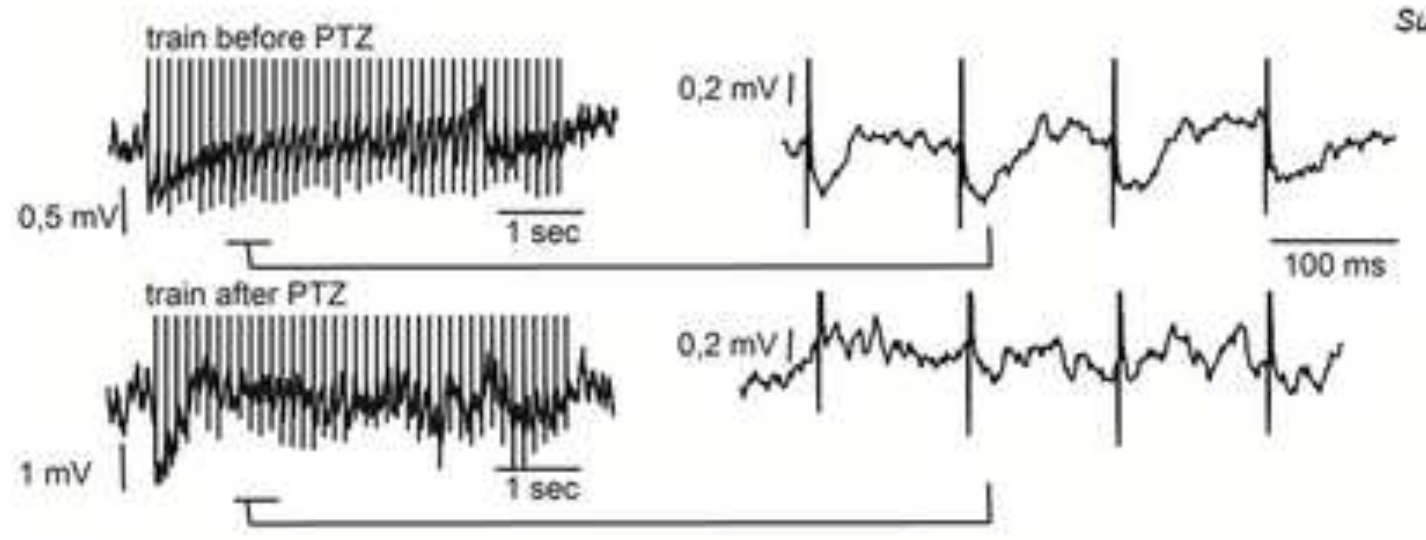

Superimposed responees

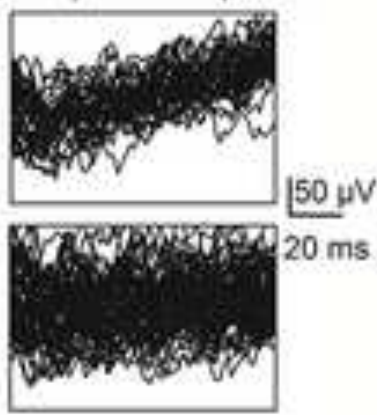

D
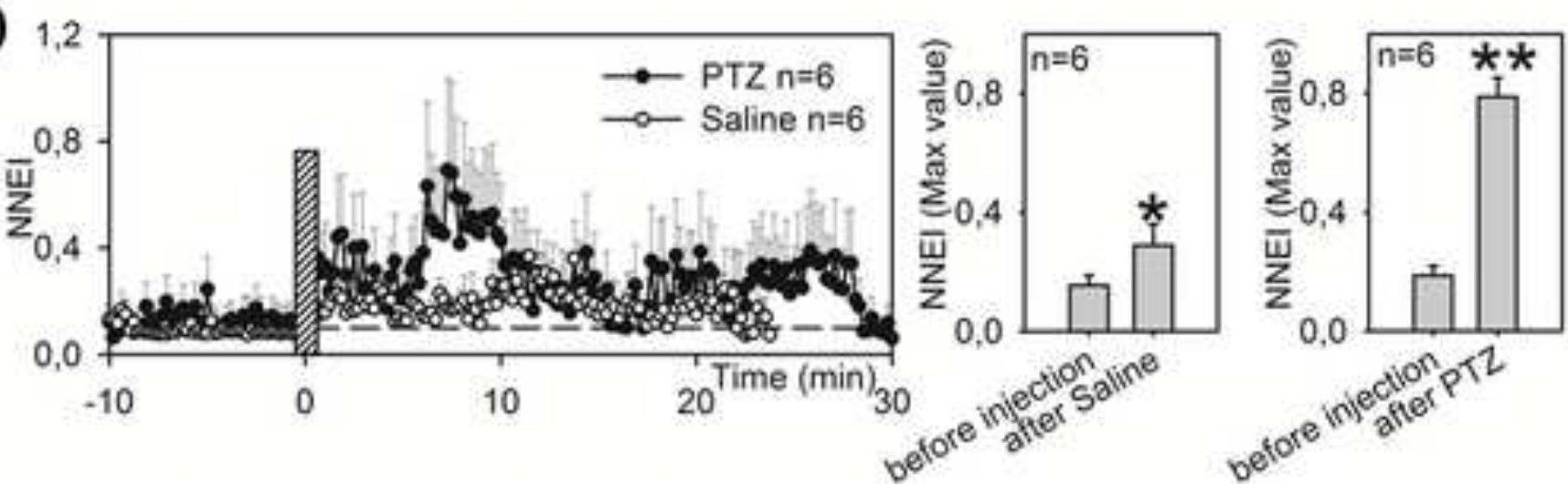
A

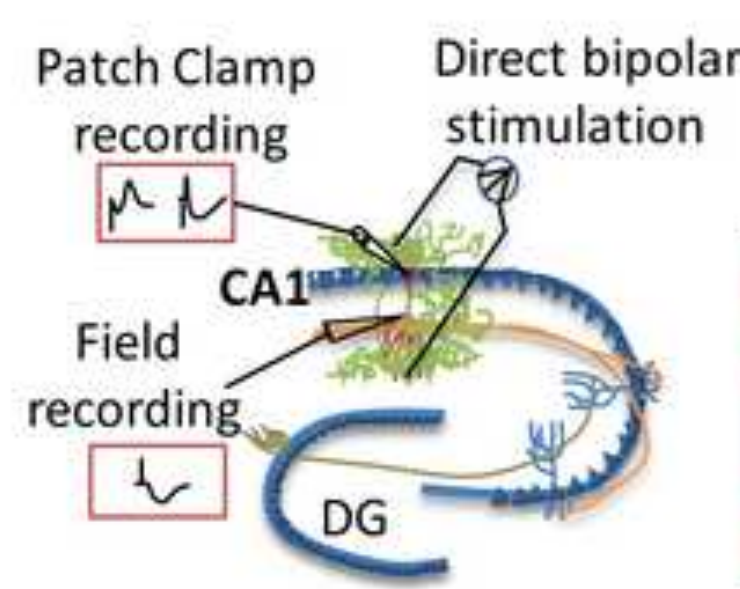

C

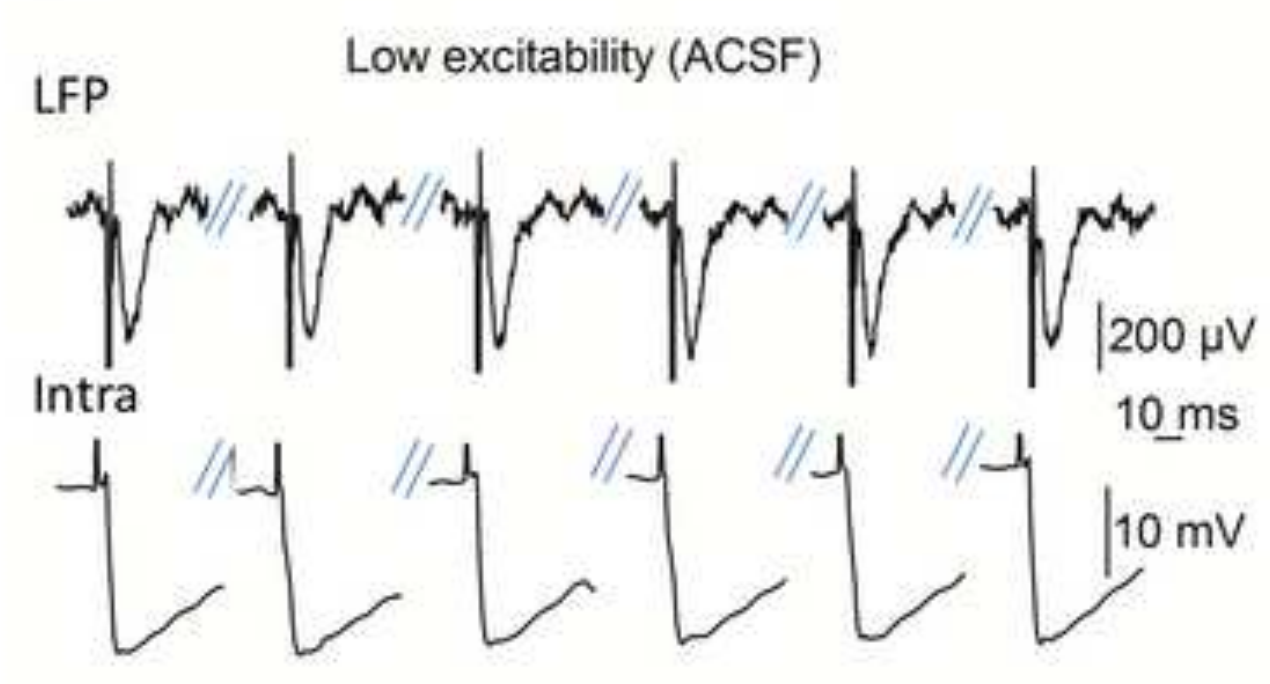

B
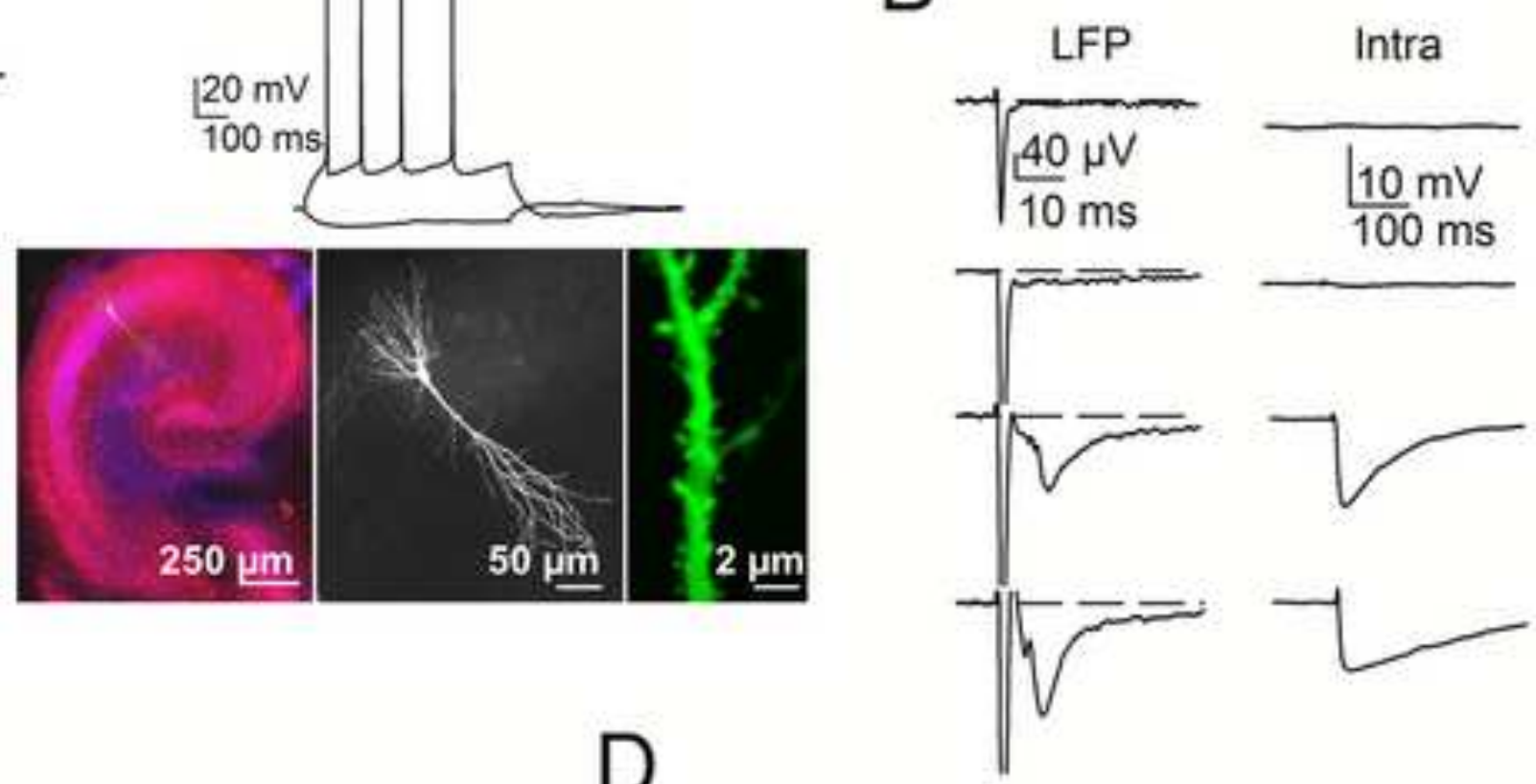

D

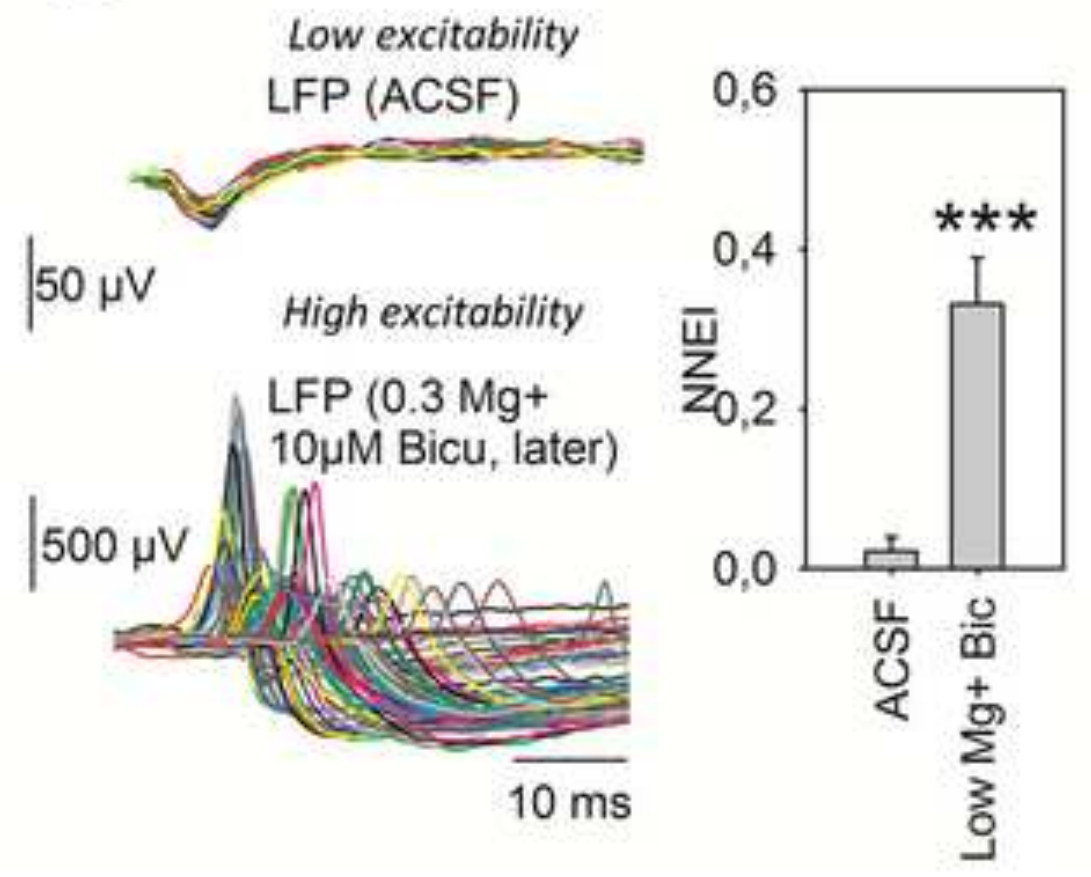


A

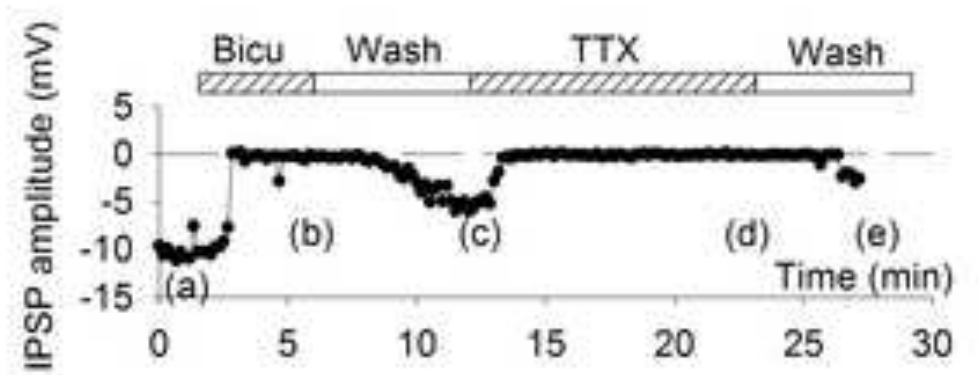

$\begin{array}{llll}\text { (a) } & \text { (b) } & \text { (c) } & \text { (d) }\end{array}$

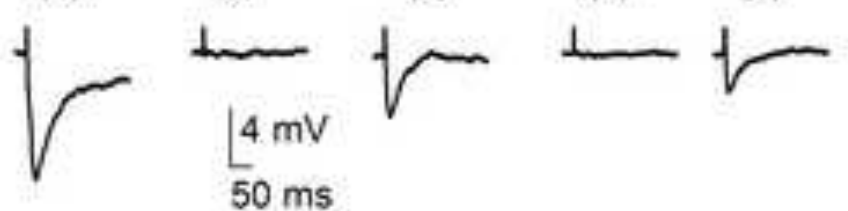

C

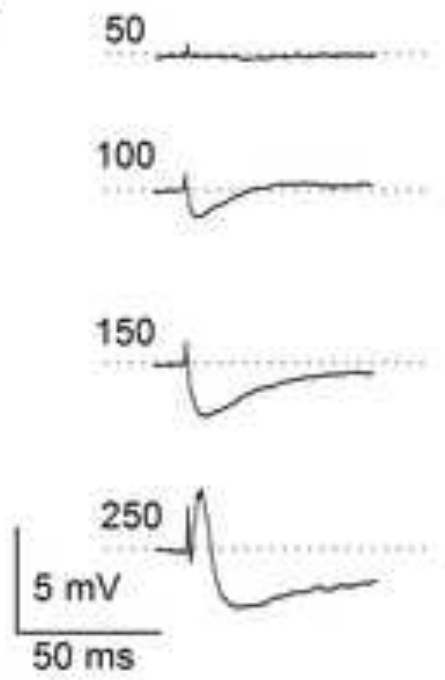

B
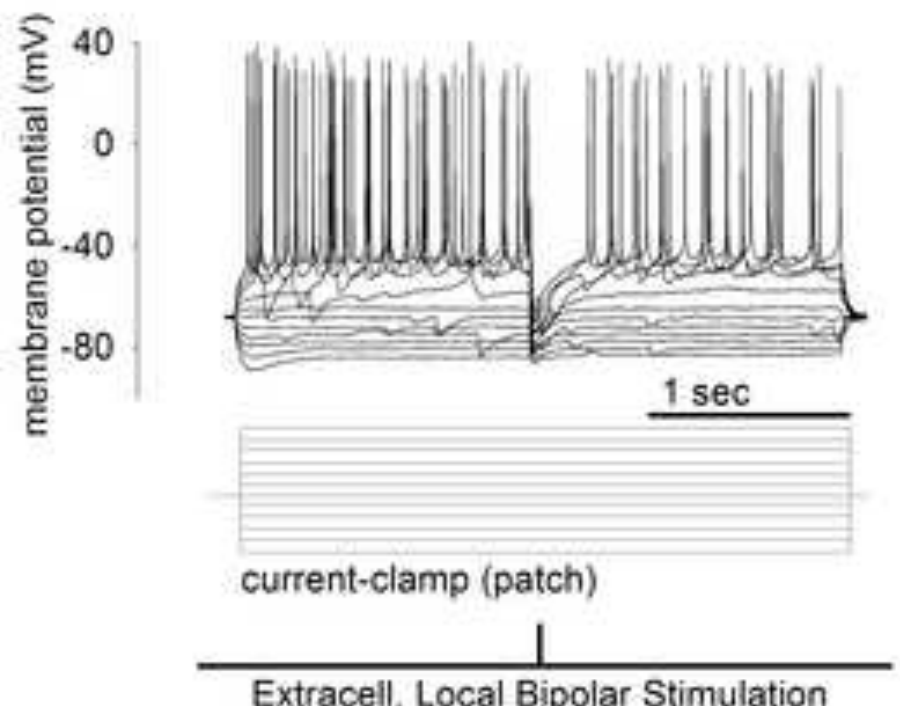

Extracell. Local Bipolar Stimulation
D

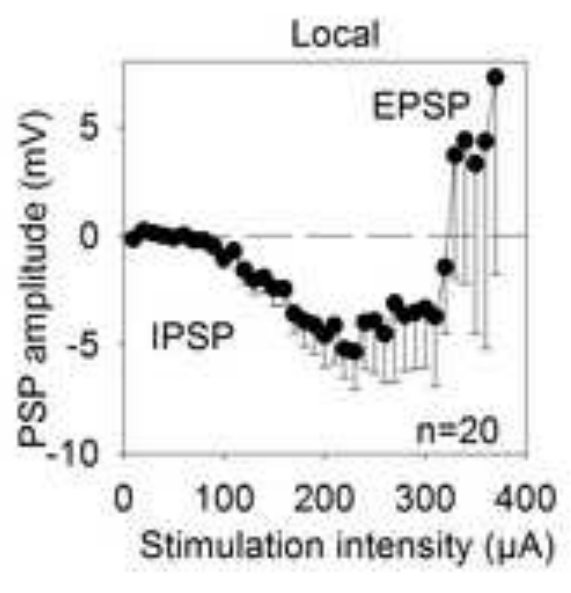

E

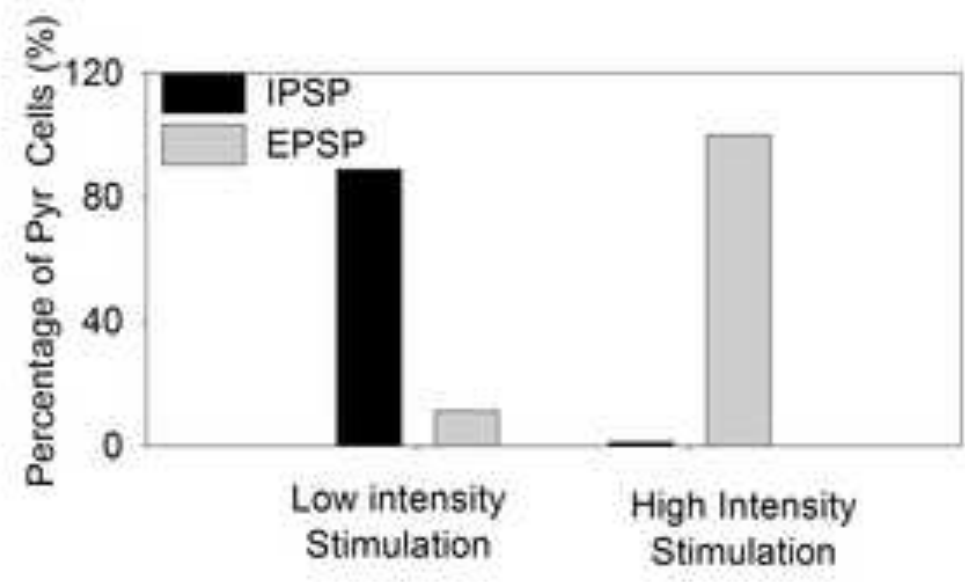




\section{Patient P1}

A

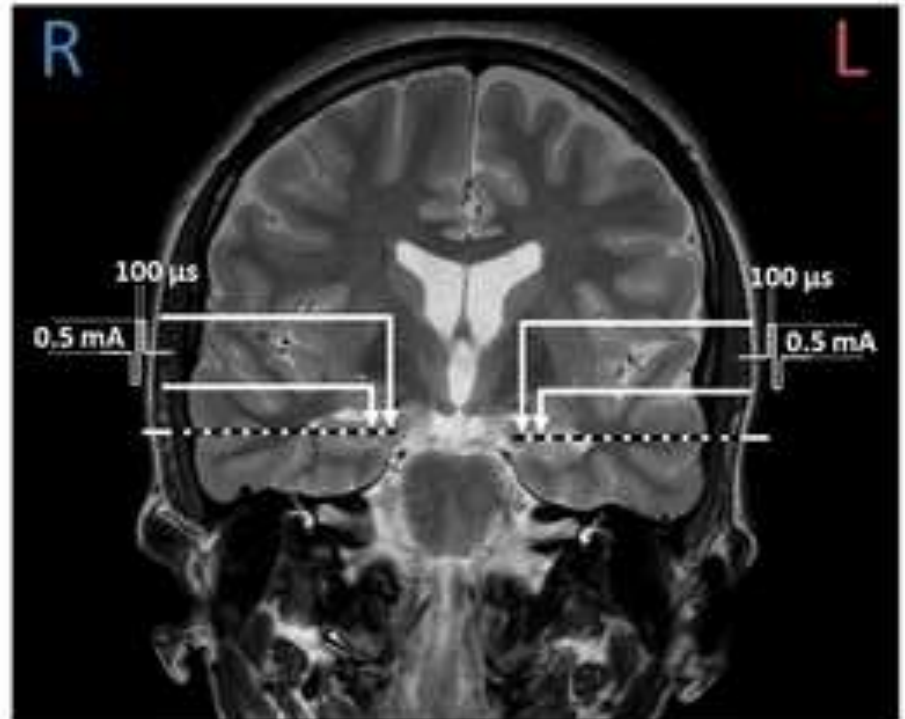

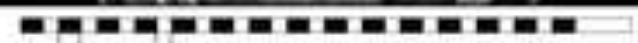

D

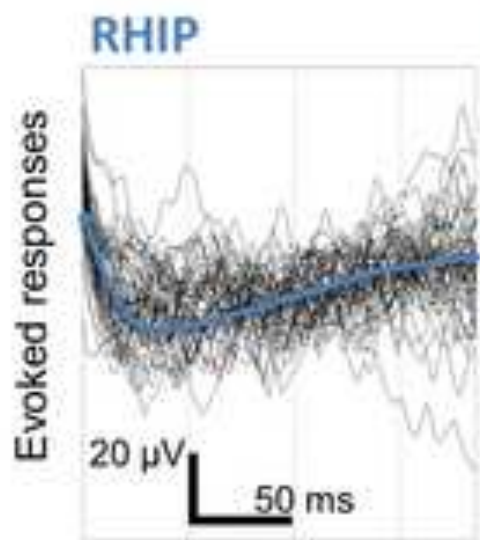

LHIP

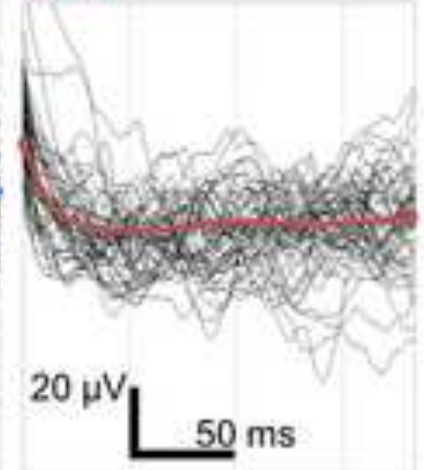

B

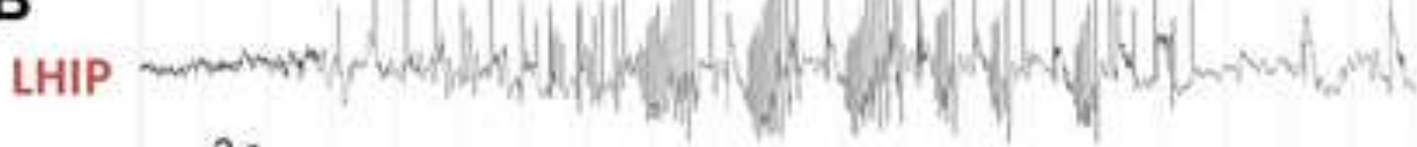

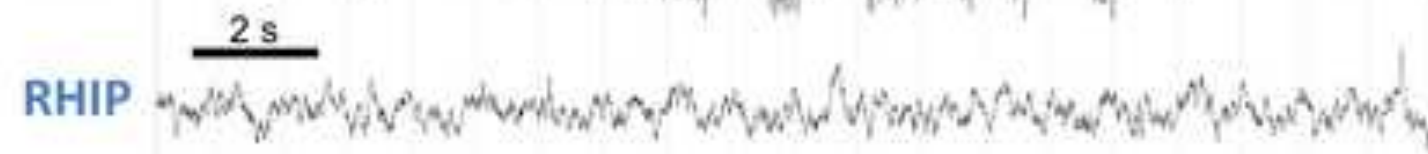

C

LHIP
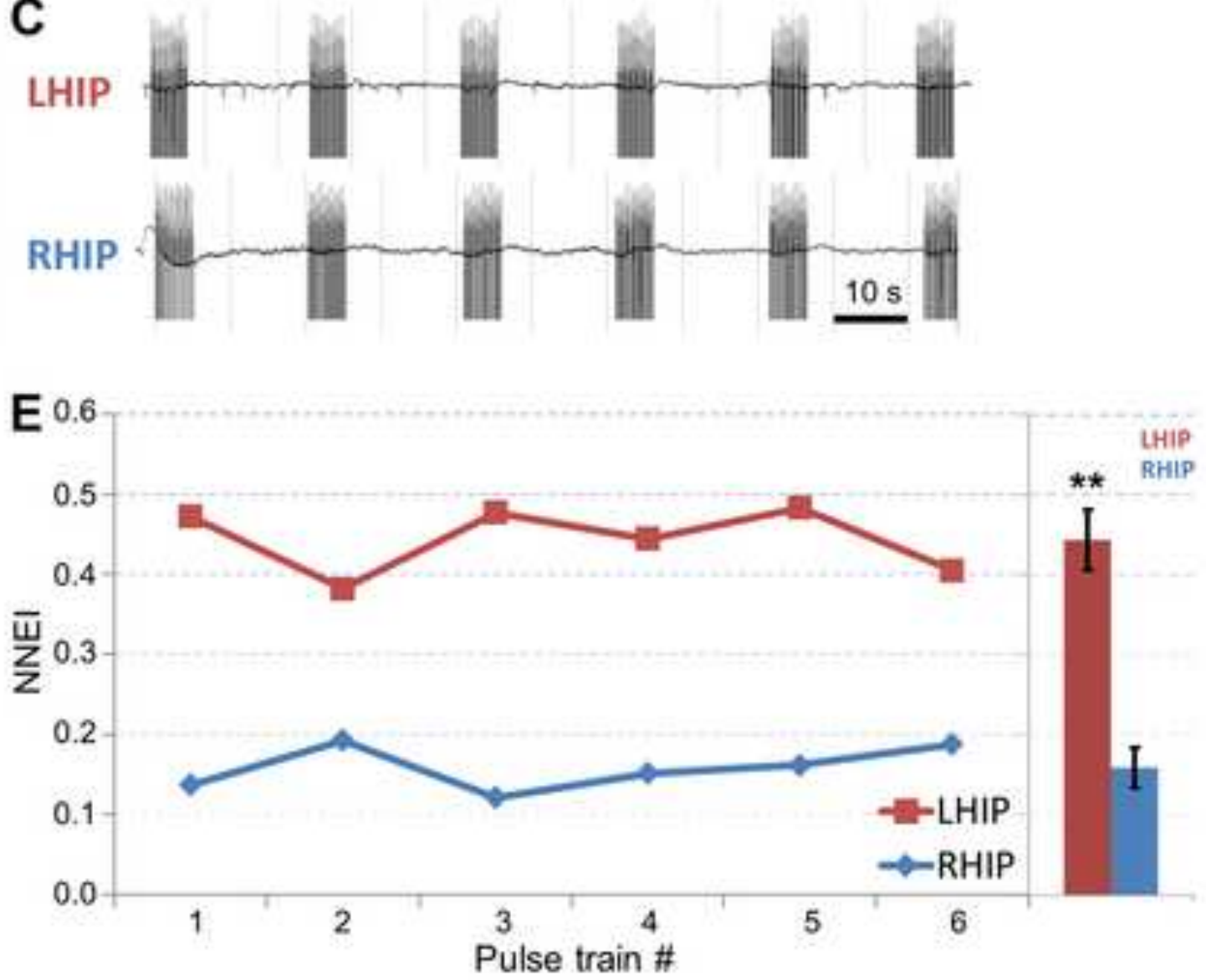


\section{A. Patient P2}

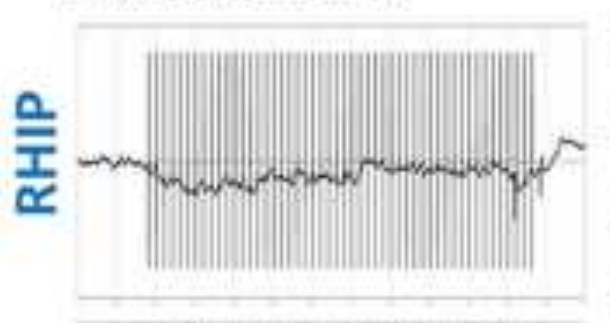

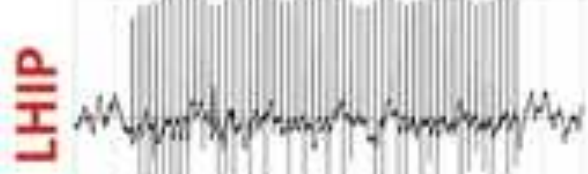

$-1 s$
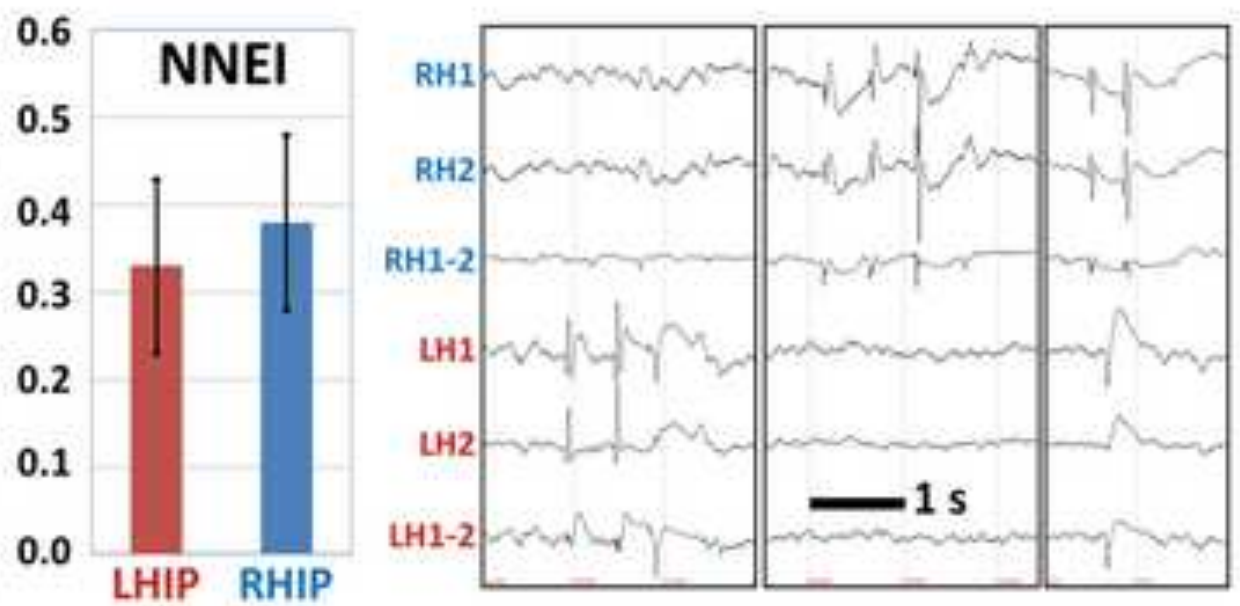

\section{B. Patient P3}

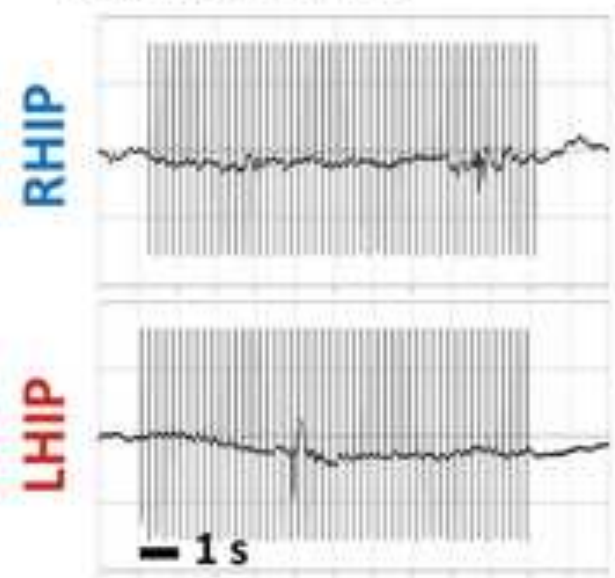

\section{Patient P4}
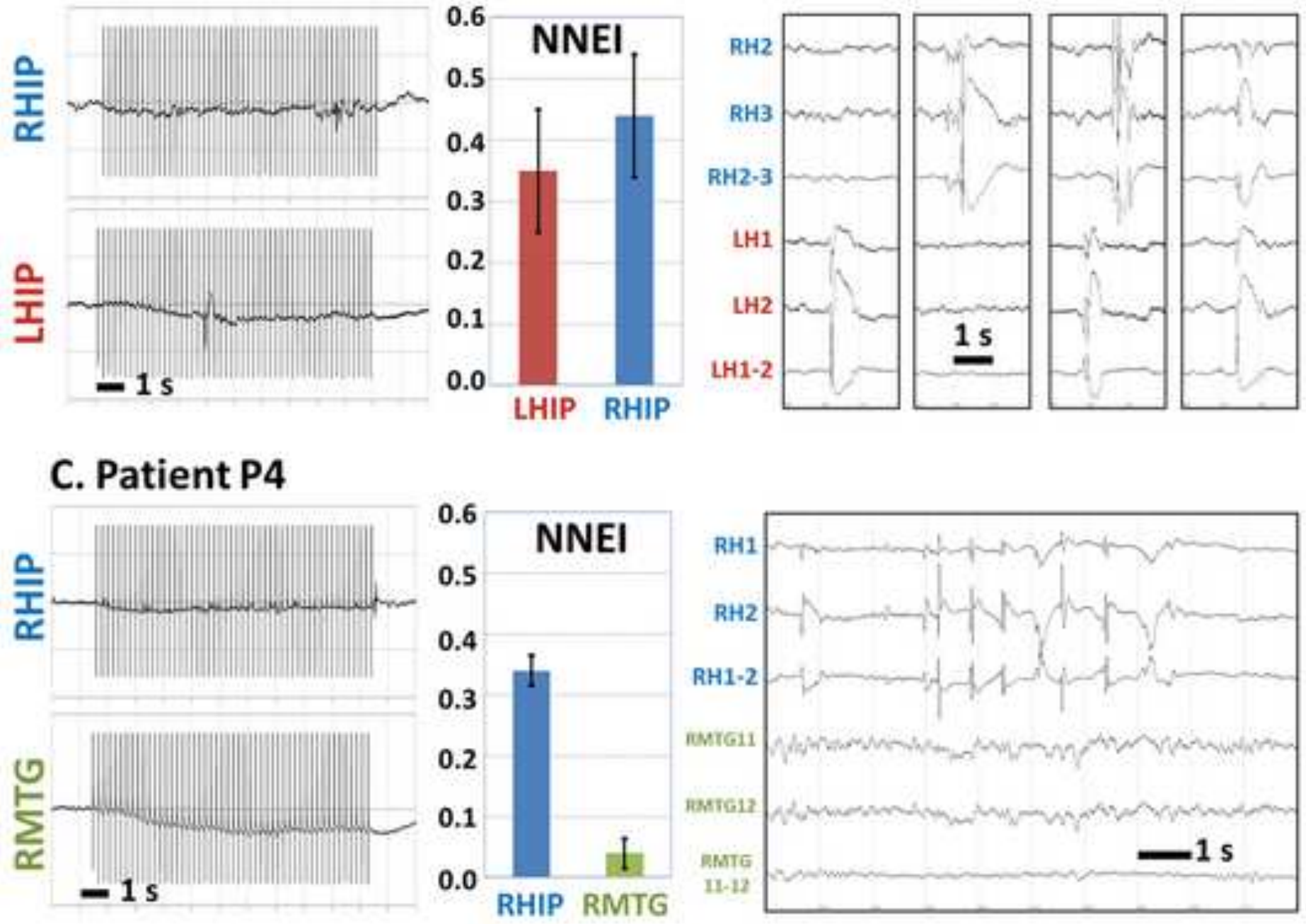


\begin{tabular}{|c|c|c|c|c|c|c|c|c|}
\hline $\begin{array}{c}\text { PATIENT } \\
\#\end{array}$ & GENDER & $\begin{array}{l}\text { AGE } \\
(y / o)\end{array}$ & $\begin{array}{l}\text { TYPE OF } \\
\text { EPILEPSY }\end{array}$ & ETIOLOGY & $\begin{array}{l}\text { SURGICAL } \\
\text { OUTCOME }\end{array}$ & $\begin{array}{c}\text { STIMULATED } \\
\text { ELECTRODE } \\
\text { CONTACTS: } \\
\text { BRAIN } \\
\text { STRUCTURE }\end{array}$ & $\begin{array}{c}\text { SEEG } \\
\text { ACTIVITY } \\
\text { (N: Normal, } \\
\text { E: } \\
\text { Epileptiform) }\end{array}$ & $\begin{array}{l}\text { STIMULATION } \\
\text { INTENSITY } \\
\text { and } \\
\text { FREQUENCY }\end{array}$ \\
\hline P1 & M & 22 & $\begin{array}{c}\text { Left } \\
\text { temporal }\end{array}$ & $\begin{array}{l}\text { Hippocampal } \\
\text { sclerosis }\end{array}$ & Engel la & $\begin{array}{l}\text { B1-2 : right } \\
\text { anterior } \\
\text { hippocampus } \\
\text { CP1-2: left } \\
\text { posterior } \\
\text { hippocampus }\end{array}$ & $\begin{array}{l}\text { B1-2: N } \\
\text { CP1-2: E }\end{array}$ & $\begin{array}{c}0.5 \mathrm{~mA} \\
10 \mathrm{~Hz}\end{array}$ \\
\hline $\mathbf{P 2}$ & $\mathrm{F}$ & 24 & $\begin{array}{l}\text { Bi } \\
\text { temporo- } \\
\text { perisylvian }\end{array}$ & Cryptogenic & $\begin{array}{c}\text { Surgery } \\
\text { contraindic } \\
\text { ation }\end{array}$ & $\begin{array}{l}\text { B1-2: right } \\
\text { anterior } \\
\text { hippocampus } \\
\text { BP1-2: left } \\
\text { anterior } \\
\text { hippocampus }\end{array}$ & $\begin{array}{l}\text { B1-2: E } \\
\text { BP1-2: E }\end{array}$ & $\begin{array}{c}0.3 \mathrm{~mA} \\
5 \mathrm{~Hz}\end{array}$ \\
\hline P3 & M & 33 & $\begin{array}{l}\text { Fronto- } \\
\text { temporal }\end{array}$ & $\begin{array}{c}\text { Probable } \\
\text { anterior left } \\
\text { focal cortical } \\
\text { dysplasia }\end{array}$ & $\begin{array}{c}\text { Surgery } \\
\text { scheduled }\end{array}$ & $\begin{array}{c}\text { B2-3: right } \\
\text { anterior } \\
\text { hippocampus } \\
\text { BP2-3: left } \\
\text { anterior } \\
\text { hippocampus }\end{array}$ & $\begin{array}{l}\text { B2-3: E } \\
\text { BP2-3: E }\end{array}$ & $\begin{array}{c}0.3 \mathrm{~mA} \\
5 \mathrm{~Hz}\end{array}$ \\
\hline P4 & $\mathrm{F}$ & 42 & $\begin{array}{c}\text { Right } \\
\text { Temporo- } \\
\text { perisylvian }\end{array}$ & $\begin{array}{l}\text { Hippocampal } \\
\text { sclerosis and } \\
\text { probable } \\
\text { anterior } \\
\text { temporal non } \\
\text { Taylor } \\
\text { associated } \\
\text { dysplasia }\end{array}$ & $\begin{array}{c}\text { Surgery } \\
\text { scheduled }\end{array}$ & $\begin{array}{l}\text { B2-3: right } \\
\text { anterior } \\
\text { hippocampus } \\
\text { C11-12: right } \\
\text { posterior } \\
\text { middle } \\
\text { temporal gyrus }\end{array}$ & $\begin{array}{c}\text { B2-3: E } \\
\text { C11-12: N }\end{array}$ & $\begin{array}{c}0.3 \mathrm{~mA} \\
5 \mathrm{~Hz}\end{array}$ \\
\hline
\end{tabular}

Table 1: Clinical data for the four patients (P1 - P4) who underwent ELBS. Clinical results are shown in figures 6 and 7. 


\begin{tabular}{|c|c|c|c|}
\hline Stimulation electrode & In vivo & In vitro & Clinical \\
\hline Type & Twisted wires & Parallel bipolar & Multi-contact \\
\hline Diameter & $139 \mu \mathrm{m}$ & $125 \mu \mathrm{m}$ & $0.8 \mathrm{~mm}$ \\
\hline Material & Stainless steel & Platinum Iridium & Platinum Iridium \\
\hline $\begin{array}{c}\text { Contact length } \\
\begin{array}{c}\text { Distance between the } \\
\text { two electrode } \\
\text { contacts }\end{array}\end{array}$ & $500 \mu \mathrm{m}$ & $400 \mu \mathrm{m}$ & $2 \mathrm{~mm}$ \\
\hline
\end{tabular}

Table 2. Technical features of electrodes used for in vivo, in vitro and clinical measurements 